\title{
Effect of Salicylic acid, Biofertilization and Sowing Dates on Peanut (Arachis hypogaea L.) Yield under Semi-arid Conditions
}

\author{
Howaida A. Maamoun and A. M. Abd El Gawad* \\ Plant Production Dept. and "Soil Fertility \& Microbiology \\ Dept., Desert Research Center, El-Matareya, Cairo, Egypt.
}

\begin{abstract}
7 WO FIELD experiments were carried out in El-Kharga Experimental 1 Farm of Desert Research Center, Al- Wadi Al- Gadeed Governorate in a sandy clay loam soil, during two summer seasons of 2010 and 2011 to study the effect of salicylic acid application, biofetilization and sowing dates on yield and its components, and the chemical composition of peanut (Giza, 6 cultivar).
\end{abstract}

The study included four levels of salicylic acid, i.e., zero salicylic acid, 100, 150 and $200 \mathrm{ppm}$ salicylic acid(SA), four different biofertilization treatments, i.e., without biofertilization (Br), Bacillus megatherium (PDB),Pseudomonas fluorescence (PS) and mixed biofertilization treatment $(\mathrm{PDB}+\mathrm{PS})$, Rhizobia was applied as base treatment and three sowing dates: April $20^{\text {th }}$, May $15^{\text {th }}$ and June $10^{\text {th }}$. The studied factors were arranged in a split- split design with four replications, where sowing date constituted the main plots, salicylic acid was arranged in the sub plots and biofertilizations were occupied sub-sub plots.

Based on yield and yield components data, it was concluded that the early sowing (April $20^{\text {th }}$ ) produced the highest significant parameters of peanut plants; plant height, weight of pods per plant, number of pods per plant, 100-seeds weight, pod and seed yields and protein and oil yields. On the reverse,the lowest values were obtained at late sown (June, $10^{\text {th }}$ ) throughout the studied two seasons (2010 and 2011). Early sown gave the chance of growing plant to complete all of its physiological processes (vegetative and reproductive processes) at proper time than that of late sown. Delaying of peanut sowing till (June, $10^{\text {th }}$ ) caused a decline in all of the studied parameters throughout the studied seasons. Increasing SA levels from 100 to $200 \mathrm{ppm}$ increased significantly all the studied parameters as compared with control in the studied two seasons. Mixed biofertilization treatment had a significant effect on yield and its components as compared with the single biofertilization treatments. Also, biofertilization treatment resulted in higher values of soil microbiological properties, i.e. total microbial counts, counts of phosphate dissolving bacterial (PDB), Pseudomonas count and nodule characteristics.

Keywords: Sowing times, Salicylic acid, Biofertilization, Heat stress conditions, Peanut . 
Desert land of the New Valley region offers opportunities for agricultural expansion. It represents $44 \%$ of the total area of Egypt covering about 3.3 million fad (Salem, 2008). However, the cultivated area does not exceed 9981 faddan, mainly occupied by field crops and depends on ground water irrigation. Peanut is a crop which offers potential for the area both for oil (40-50\%) and stock feed (30-50\% proteins) (Hatam \& Abbasi, 1994). There is a need for optimization of the peanut production system in this desert land area particularly with respect to possible improvement of the microbiological environment.

A Significant increases in peanut yield can be achieved by early or late of planting (Baldwin, 2005), with a previous study indicating that early planting produced $20 \%$ to $50 \%$ greater pod yields than late planting (Naab et al., 2004; Laurence, 1983: Mozingo et al., 1991 and Bala et al., 2011). The yields of plant components (pod and seeds) and their respective rates, as well as flower, peg and pod numbers were significantly affected by planting date (Kasai et al., 1999). Frimpong (2004) reported that plant height, biomass, and pod yield were significantly affected by planting date and environmental factors.

Growth regulators are one of the most important factors for yield improvement in various field crops (Ali \& Mahmoud, 2012). Salicylic acid, (SA) (2hydroxybenzoic acid) as a natural plant hormone has many effects on physiological processes and growth plants (Khan et al., 2003) and development and plays an active role in plant defense responses (Janda et al., 2007). Exogenously added SA also increased the heat tolerance of mustard (Dat et al., 2000). It occurs naturally in plants in low amounts and has been shown to promote nutrient uptake, chlorophyll synthesis, photosynthesis and protein synthesis (Khan et al., 2003 and Sakhabutdinova et al., 2003). Salicylic acid may be a prerequisite for the synthesis of auxin and / or cytokinin (Metwally et al., 2003). Furthermore, SA has an important role in tolerance of some environmental stresses such as heat, salts and drought stress (Khan et al., 2003 and Hussein et al., 2007). Foliar spray of salicylic acid as a $100 \mathrm{ppm}$ concentration increased number of pod/plant, number of seed /pod, seed weight/plant and seed green gram yield/ ha (Sujatha, 2001). Naturally, most sandy soils are low in organic matter and nutrient content that means adding foliar fertilizers is much more essential to plants which will be cultivated in the last few decades, foliar fertilizers are used not only to give great yields or to improve the quality of crops but also to compensate the deficient soil nutrients, particularly in sandy soils due to the vertical expansion in agriculture (Ali \& Mahmoud, 2012).

Biofertilizer, an alternative source of $\mathrm{N}$-fertilizer, especially rhizobia in legume symbiosis is an established technology. Use of the biofertilizers can also prevent the depletion of the soil organic matter (Jeyabal \& Kuppuswamy, 2001). Inoculation with bacterial biofertilizer may reduce the application of fertilizer-N by increasing N uptake by plants (Choudhury \& Kennedy, 2004; Kennedy et al., 2004 and Mia et al. 2005, 2007). Nitrogen fixation and plant growth enhancement by rhizosphere bacteria might be important factors for achieving a sustainable agriculture in the future (Baset Mia \& Shamsuddin, 2010)

Egypt. J. Agron. 35, No. 1 (2013) 
Soil microorganisms can fix atmospheric nitrogen, soluble phosphate, affect plant growth through synthesis of growth promoting substances and enhance the decomposition of plant residues to release vital nutrients and increase humic content in soils. Thus addition of these to cropping soils may be an environmentally benign approach for improved nutrient management and ecosystem function (Wu et al., 2005 and Kaci et al., 2005). Possible options for improving peanut nutrition include Bradyrhizobium ( $\mathrm{N}$ nutrition) and Pseudomonas (P nutrition). Peanut (Arachis hypogaea L.), Family Leguminosae is usually nodulated by rhizobia of the genus Bradyrhizobium as demonstrated by Van Rossum et al. (1995): Rhizobia are symbiotic bacteria which fix atmospheric nitrogen into ammonia. Phosphate solubilizing bacteria have the ability to increase the available phosphorous for plant through production of organic acids (Mehana \& Farag, 2000). The microorganisms involved in $\mathrm{P}$ solubilisation can enhance plant growth by increasing the efficiency of biological nitrogen fixation, enhancing the availability of other trace elements and by production of plant growth promoting substances (Gyaneshwar et al., 2002). Fluorescent Pseudomonas spp. strain 267 promoted growth of different plants under field conditions and enhanced symbiotic nitrogen fixation in clover (Marek-Kozaczuk \& Skorupska, 1997).

Therefore, the present work aimed to extend investigate the existing information on the benefits of early sowing to study the effects of levels of salicylic acid and types of biofertilization and their interactions with sowing dates on yield, yield components and chemical composition of peanut plants grown under New Valley conditions of Egypt.

\section{Materials and Methods}

Two field experiments were carried out at the Agricultural Experimental Farm of the Desert Research Center at El-Kharga Oasis, New Valley Governorate of Egypt. The major portion the El-Kharga Oasis site is between longitudes $28^{\circ} 48^{\circ}$ and $29^{\circ} 21^{-} \mathrm{E}$ and latitudes $25^{\circ} 28^{-}$and $25^{\circ} 44-\mathrm{N}$ ). The experiments were carried out during two successive summer seasons of 2010 and 2011 to study the response of peanut, cv., (Giza, 6) to sowing dates (April $20^{\text {th }}$, May $15^{\text {th }}$ and June $10^{\text {th }}$ ) and salicylic acid foliar application (zero salicylic acid, 100, 150 and $200 \mathrm{ppm}$ ) and four different biofetilization treatments (Bradyrhizobium as base for all treatments, Bacillus megatherium (PDB), Pseudomonas fluorescence (PS) and mixed biofertilization treatment (PDB+PS). The physical and chemical properties of the soil before cultivation are presented in Table 1 , chemical analysis of irrigation water in Table 2 and temperature of the site 2010 and 2011 according to Meteorological Station at New Valley in Egypt in Table 3. 
TABLE 1. Some Physical and Chemical characteristics of the studied soil .

\begin{tabular}{|c|c|c|c|c|c|c|c|c|c|c|}
\hline \multicolumn{11}{|c|}{ Mechanical analysis } \\
\hline \multicolumn{3}{|c|}{ Sand } & \multicolumn{3}{|c|}{ Clay } & \multicolumn{2}{|c|}{ Silt } & \multicolumn{3}{|c|}{ Soil texture } \\
\hline \multicolumn{3}{|c|}{$51.5 \%$} & \multicolumn{3}{|c|}{$30.4 \%$} & \multicolumn{2}{|c|}{$18.1 \%$} & \multicolumn{3}{|c|}{ Sandy clay loam } \\
\hline \multicolumn{11}{|c|}{ Chemical analysis } \\
\hline \multirow[t]{2}{*}{ pH } & \multirow{2}{*}{$\begin{array}{c}\text { EC } \\
\text { ds } / \mathbf{m l}\end{array}$} & \multirow[t]{2}{*}{ T.N } & \multicolumn{4}{|c|}{ Cations (me/l) } & \multicolumn{4}{|c|}{ Anions (meq/l) } \\
\hline & & & $\mathrm{Ca}^{+2}$ & $\mathrm{Mg}^{+2}$ & $\mathbf{K}^{+}$ & $\mathrm{Na}^{+}$ & $\mathrm{CO}_{3}{ }^{-2}$ & $\mathrm{HCO}^{-3}$ & $\mathrm{Cl}^{-}$ & $\mathrm{SO}_{4}{ }^{-2}$ \\
\hline 8.32 & 24.8 & $100 \mathrm{ppm}$ & 4.00 & 3.25 & 1.66 & 15.79 & 0.00 & 1.2 & 18.0 & 5.5 \\
\hline \multicolumn{11}{|c|}{ Trace elements (ppm) } \\
\hline \multicolumn{2}{|c|}{$\mathbf{Z n}$} & \multirow{2}{*}{\multicolumn{2}{|c|}{ Mn }} & \multicolumn{2}{|c|}{$\mathbf{C u}$} & \multicolumn{2}{|c|}{$\mathrm{Fe}$} & \multicolumn{3}{|c|}{ B } \\
\hline \multicolumn{2}{|c|}{5.97} & & & \multicolumn{2}{|c|}{1.79} & \multicolumn{2}{|c|}{33.08} & \multicolumn{3}{|c|}{0.56} \\
\hline
\end{tabular}

TABLE 2. The chemical analysis of irrigation water .

\begin{tabular}{|c|c|c|c|c|c|c|}
\hline $\mathbf{p H}$ & $\mathbf{E C d s} / \mathbf{m l}$ & Units & $\mathbf{C a}^{+2}$ & $\mathbf{M g}^{+2}$ & $\mathbf{N a}^{+}$ & $\mathbf{K}^{+}$ \\
\hline 7 & 676 & $\mathrm{ppm}$ & 31.89 & 10.80 & 1.33 & 19.40 \\
\hline & Units & $\mathrm{CO}_{3}{ }^{-2}$ & $\mathrm{HCO}_{3}^{-}$ & $\mathrm{SO}_{4}{ }^{-2}$ & $\mathrm{Cl}^{-}$. \\
\cline { 3 - 7 } & & $\mathrm{ppm}$ & 0.00 & 64.06 & 30.87 & 36.97 \\
\hline
\end{tabular}

TABLE 3. Temperature of the site 2010 and 2011(Meteorological Station at New Valley in Egypt).

\begin{tabular}{|c|c|c|c|c|c|c|}
\hline \multirow{2}{*}{ Month } & \multicolumn{3}{|c|}{$\begin{array}{c}\text { Mean temperatures ( } \mathrm{C} \text { ) } \\
2010\end{array}$} & \multicolumn{3}{|c|}{$\begin{array}{c}\text { Mean temperatures ( } \mathrm{C} \text { ) } \\
2011\end{array}$} \\
\hline & Max & Min & Mean & Max & Min & Mean \\
\hline April $1^{s t}-10^{\text {th }}$ & 34.71 & 18.41 & 26.52 & 30.23 & 13.49 & 22.53 \\
\hline April $11-20^{t h}$ & 37.28 & 18.93 & 28.78 & 32.91 & 16.83 & 24.89 \\
\hline April $21-30^{\text {th }}$ & 34.65 & 18.85 & 27.17 & 34.59 & 18.20 & 26.81 \\
\hline May $1^{\text {st }}-10^{\text {th }}$ & 37.58 & 18.31 & 28.99 & 36.90 & 20.28 & 28.93 \\
\hline May $11-20^{\text {th }}$ & 39.13 & 22.88 & 31.14 & 35.02 & 19.67 & 27.98 \\
\hline May $21-31^{s t}$ & 38.16 & 23.56 & 30.29 & 39.97 & 24.66 & 32.77 \\
\hline June $1^{\text {st }}-10^{\text {th }}$ & 40.34 & 24.59 & 33.16 & 39.64 & 26.78 & 33.22 \\
\hline June $11-20^{\text {th }}$ & 41.44 & 25.49 & 30.44 & 38.68 & 23.82 & 31.77 \\
\hline June $21-30^{\text {th }}$ & 42.98 & 26.29 & 35.32 & 38.95 & 24.35 & 32.02 \\
\hline July & 40.47 & 26.56 & 34.28 & 42.02 & 25.87 & 34.56 \\
\hline August & 42.14 & 26.33 & 35.14 & 40.63 & 24.58 & 33.31 \\
\hline September & 39.39 & 24.59 & 31.88 & 37.83 & 21.38 & 30.51 \\
\hline October & 37.22 & 21.69 & 29.53 & 33.28 & 19.99 & 26.38 \\
\hline
\end{tabular}

Assay of biochemical activities in vitro

The ability of the tested microbial isolates to produce biochemical activities was evaluated under in vitro conditions, through determination of their efficiency to solubilize phosphate (Nautiyal, 1999), produce of growth regulators (Rizzolo et al., 1993), carry out nitrogen fixation (Scholhorn \& Burris, 1967), produce enzymes (Barrow \& Veltham, 1993), produce antibiotics (Jarlier et al., 1996) and produce total carbohydrate (Cherry, 1973).

Egypt. J. Agron. 35, No. 1 (2013) 
Peanut (Arachis hypogace L.) cv., Giza 6 was inoculated just before sowing with the specific bacterial inoculants. Seeds of peanut were sown on three sowing dates (April, 20 $0^{\text {th }}$, May, $15^{\text {th }}$ and June 10 ${ }^{\text {th }}$ ) with two successive seasons 2010 and 2011.

Seeds of peanut were washed and immersed for $30 \mathrm{~min}$ in liquid culture of the specific bacteria to be tested. Carboxymethyl cellulose (CMC 0.5\%) was used as an adhesive agent. Seeds were then dried at room temperature for two hours. Fresh liquid cultures $48 \mathrm{hr}$ old containing pure local strains of Bradyrhizobium (Br), Bacillus megatherium var. phosphaticm (PDB) and Pseudomonas fluorescens (PS), previously isolated from the rhizosphere of soils of New Valley region were used. These had been purified and identified according to Bergey's Manual (1984). They were used as biofertilizers in the form of single and mixed inoculations at the rate of $\sim 10^{8} \mathrm{cfu} / \mathrm{ml}$.

Plants were sprayed with distilled water (control), 100, 150 and $200 \mathrm{ppm}$, of salicylic acid dissolved in distilled water. All solutions were sprayed to the shoots uniformly using a hard-pump sprayer.

Normal cultural practices for peanut were applied as recommended in the district. The experimental design was split-split plot with four replicates: three sowing dates were arranged in the main plots, salicylic acid as foliar application treatments were assigned in the sub plots and biofertilization treatments were allocated in the sub- sub plots. The plot area was $10.5 \mathrm{~m}^{2}$ consisting of five rows ( $3.5 \mathrm{~m}$ length and $60 \mathrm{~cm}$ between rows). Plants were thinned to a single plant per hill and the distance between hills was $25 \mathrm{~cm}$ apart.

Foliar spraying with salicylic acid was done twice, firstly during the vegetative stage (35 days after sowing) and then at pod development ( 75 days after sowing) at the rate of $400 \mathrm{~L} / \mathrm{fad}$.

Peanut plants were harvested at mid September, first of October and end of October in the two studied growing seasons, respectively for the three sowing dates. Ten guarded and competitive plants of the middle two rows were taken randomly to determine the yield components as follows: - plant height $(\mathrm{cm})$, weight of pods per plant $(\mathrm{g})$, number of pod per plant, and 100-seed weight $(\mathrm{g})$. Whole plant was uprooted and the pods were air dried to weigh and calculate seed yield per Faddan.

Seeds content of $\mathrm{N}$ was determined using the methods described by Chapman \& Pratt (1978) and protein content calculated by multiplying N\% by 6.25 . Seed oil content was determined according to A.O.A.C. (1980).

Soil samples of the peanut rhizosphere were collected in both seasons and analyzed for microbial determinations. Nodulation was determined by nodule numbers and dry wt. of nodule. Total microbial counts were determined on Bunt and Rovira medium (Nautiyal, 1999) using the decimal plate method technique. 
Counting the number of growing phosphate dissolving bacteria was carried out using Bunt and Rovira medium after addition of $5 \mathrm{ml}$ sterile solution of $10 \%$ of $\mathrm{K}_{2} \mathrm{HPO}_{4}$ and of $10 \mathrm{ml}$ of sterile solution of $10 \% \mathrm{CaCl}_{2}$ to each $100 \mathrm{ml}$ of the medium. The most probable number (MPN) of Pseudomonades was determined after incubating the tubes at $30 \pm 2{ }^{\circ} \mathrm{C}$ for $48 \mathrm{hr}$ on King's B medium (King et al, 1954). Estimates of number of pseudomonades by MPN technique were calculated using Cochran's table. Nodulation (nodule number and dry weight of nodule) was determined by the method of Wilson \& Reisenauer (1963).

The data were subjected to analysis of variance of split- split plot designs according to the method described by Gomez \& Gomez (1984). Least significant differences (LSD) at the 5\% level were used to compare the means of tested treatments.

\section{Results and Discussion}

\section{Biochemical activities of microbial isolates}

Table 4 shows the biochemical activities of the Bradyrhizobium japonicum, Bacillus megatherium and Pseudomonas fluorescens used in the trial for production of hormones, antibiotic, enzymes, phosphate solubilization, nitrogen fixation and total carbohydrates production. Hormonal activities, enzyme production and phosphate solubilization are common features of all the tested microorganisms. However, B. megatherium appeared to be superior to the other isolates in phosphate solubilization. Only Bradyrhizobium showed nitrogenase activity on other side $P$. fluorescens and B. megatherium showed antimicrobial activity. Bradyrhizobium showed highest indole acetic acid production (IAA) while Pseudomonas gave highest cytokinine production as expected the microorganisms exhibited the types of biochemical and hormonal activity in vitro that could result, in beneficial action in the field (Van Rossum et al. 1995; Vargas et al., 2009; Verma et al., 2010 and El- Saidy \& Abd El-Hai, 2011).

TABLE 4. Biochemical activities of microbial isolates .

\begin{tabular}{|c|c|c|c|c|c|c|c|c|}
\hline \multirow[t]{2}{*}{ Isolate } & \multirow[t]{2}{*}{$\begin{array}{l}\text { Nitrogenase } \\
\mu_{1} C_{2} H_{4} H^{-1} I^{-1}\end{array}$} & \multicolumn{3}{|c|}{$\begin{array}{c}\text { Hormonal activity } \\
\text { Quantitative (HPLC) / } \\
\mu \mathrm{g} / \mathrm{ml}\end{array}$} & \multicolumn{4}{|c|}{ Enzyme activity* } \\
\hline & & IAA & $\mathbf{G A}_{3}$ & Cytokinin & Amylase & Cellulase & Protease & Pectinase \\
\hline B.japonicum & 463 & 1.8 & 2.5 & 8.7 & + & + & + & + \\
\hline B.megatherium & - & 0.24 & 1.32 & 10.2 & + & + & + & + \\
\hline P.fluorescens & - & 0.91 & 2.19 & 14.15 & - & + & + & + \\
\hline \multirow[t]{2}{*}{ Isolate } & \multirow{2}{*}{$\begin{array}{l}\text { P.solubilizat- } \\
\text { ion mg p/l }\end{array}$} & \multicolumn{3}{|c|}{ Antibiotic production (mm) } & \multicolumn{4}{|c|}{ Total carbohydrates (mg/l) } \\
\hline & & \begin{tabular}{|l|}
$E$. \\
coli
\end{tabular} & $\begin{array}{l}F . \\
\text { oxysporum }\end{array}$ & \begin{tabular}{|l} 
R. \\
solani
\end{tabular} & Mono & Di & Poly & Total \\
\hline B.japonicum & 2.85 & - & - & - & 257 & 114.68 & 12.2 & 383.7 \\
\hline B.megatherium & 4.75 & 21 & 26 & 19 & 108 & 74.31 & 56.27 & 238.58 \\
\hline P.fluorescens & 1.1 & 24 & 31 & 36 & 91 & 63.81 & 17.39 & 172.2 \\
\hline
\end{tabular}

* for enzyme activity; + indicates existence of activity, - indicates no activity. 


\section{Effect of treatments on the yield of peanut and its components}

Table 5, indicates that in the two growing seasons, the yield of peanut and its attributes; i.e., plant height $(\mathrm{cm})$, weight of pods per plant $(\mathrm{g})$, number of pods per plant, 100 -seeds weight $(\mathrm{g})$, pod and seed yields ( $\mathrm{kg} / \mathrm{fad})$ and protein and oil yields $(\mathrm{kg} / \mathrm{fad})$, exhibited significant differences for different dates of sowing. The highest increases for all parameters were recorded for early sown (April $20^{\text {th }}$ ), while, late sown (June $10^{\text {th }}$ ) show the lowest values (Table 5). Among different rates of salicylic acid and biofetilization treatments there were significant variations at the level $(p<0.05)$. This may be due to the efficiency of the sink than the source under early sowing date. The results are in agreement with Ahmed et al. (2007) who reported that late sown crop failed to mature and yielded $15-25 \%$ production less than the early sowing. The late crop had a shorter period for the production of pods and also a slightly lower rate of pod production causing a reduction in seeds and weight of pod/plant was similar at two sowing. Laurence (1983), reported that late sowing reduced pod yield by $19 \%$ (from 5.02 to 4.21 ton/ha) and seeds yield by $18 \%$ (from 3.55 to 2.85 ton/ha) compared with early sowing. Bell (2001), reported that commercial pod yield being decreased by 28 and $31 \%$, respectively, for the final sowing date $\left(\right.$ June $\left.10^{\text {th }}\right)$.

Sowing peanut in $20^{\text {th }}$ of April exerted percentage increase in seeds yield over these cultivated in $15^{\text {th }}$ of May and $10^{\text {th }}$ of June by 9.95 and $42.48 \%$ in 2010 season, and by 7.69 and $44.27 \%$ in 2011 season, respectively.

As respect to protein and oil yields ( $\mathrm{kg} / \mathrm{fad}$.), of peanut seeds were decreased by delaying sowing date from April $20^{\text {th }}$ to June $10^{\text {th }}$ in both studied seasons. Delaying sowing date from April $20^{\text {th }}$ to May $15^{\text {th }}$ and June $10^{\text {th }}$ decreased accumulative heat units by 0.72 and $13.21^{\circ} \mathrm{C}$ in first season and by 13.96 and $25.08{ }^{\circ} \mathrm{C}$ in the second season comparing with April $20^{\text {th }}$, respectively (Table 3 ). Increasing protein and oil yields by sowing peanut seeds early may be due to environmental conditions in this period which seem to be suitable for translocation the nutrient to seed and consequently increasing 100-seed weight and seeds production as well as oil yields. (Wahid et al., 2007 and Bala et al., 2011).

Data presented in Table 5, clearly showed that salicylic acid treatments significantly $(\mathrm{p}<0.05)$ increased all the studied parameters; i.e., plant height $(\mathrm{cm})$, weight of pods per plant (g), number of pods per plant, 100-seeds weight (g), pod and seed yields $(\mathrm{kg} / \mathrm{fad})$ and protein and oil yields $(\mathrm{kg} / \mathrm{fad})$ than untreated plants during 2010 and 2011. The data also indicated that the effect of SA at $200 \mathrm{ppm}$ concentration was more pronounced than other concentrations as well as the control. The highest values of plant height $(\mathrm{cm}), 100$-seed weight $(\mathrm{g})$, seed, protein and oil yields $(\mathrm{kg} / \mathrm{fad})(41.5,85.1,473.6,87.3$ and 174.6 in the 2010) and $(42.4,85.8,481.6$, 99.9 and 181.5 in the 2011) respectively, were registered at concentration of $200 \mathrm{ppm}$ salicylic acid. These results may be due to the role of SA in enhancing some physiological and biochemical aspects (Ali \& Mahmoud, 2012). The stimulation effect of SA on yield and its components was confermed by El-Shraiy \& Hegazi (2009) on pea plant and the beneficial effect of spraying with SA could be attributed to its vital role activity in the function of enzymes in various plants which led to 
increase in yield components (Khan et al., 2003 and Shu \& Hui, 2008). In this concern Amutha et al. (2007) stated that foliar application with SA improved the productivity of crop plants under moderately high temperature. The associative action of salicylic acid led to a significant increase in oil yield as compared to salicylic alone.

TABLE 5. Main effects of sowing date, salicylic acid and biofertilization on yield and its components of peanut in the two growing seasons .

\begin{tabular}{|c|c|c|c|c|c|c|c|c|}
\hline Treatment & $\begin{array}{l}\text { Plant } \\
\text { height } \\
(\mathrm{cm})\end{array}$ & $\begin{array}{l}\text { Weight } \\
\text { of } \\
\text { pods/ } \\
\text { plants } \\
\text { (g) }\end{array}$ & $\begin{array}{c}\text { No. } \\
\text { of pods/ } \\
\text { plants }\end{array}$ & $\begin{array}{c}100- \\
\text { seeds } \\
\text { weight } \\
\text { (g) }\end{array}$ & $\begin{array}{c}\text { Pod } \\
\text { yield } \\
(\mathrm{kg} / \mathrm{fad})\end{array}$ & $\begin{array}{c}\begin{array}{c}\text { Seed } \\
\text { yield }\end{array} \\
\text { (kg/fad) }\end{array}$ & $\begin{array}{c}\text { Protein } \\
\text { yield } \\
\text { (kg/fad) }\end{array}$ & $\begin{array}{l}\text { Oil yield } \\
\text { (kg/fad) }\end{array}$ \\
\hline \multicolumn{9}{|c|}{2010 Season } \\
\hline \multicolumn{9}{|c|}{ Sowing date } \\
\hline $20^{\text {th }}$ April & 43.34 & 40.01 & 29.10 & 88.62 & 845.17 & 445.42 & 90.3 & 177.7 \\
\hline $15^{\text {th }}$ May & 38.16 & 38.66 & 28.27 & 86.24 & 773.19 & 401.10 & 64.7 & 136.7 \\
\hline $10^{\text {th }}$ June & 34.83 & 37.22 & 26.50 & 69.37 & 562.10 & 256.17 & 40.0 & 74.3 \\
\hline LSD5\% & 0.22 & 0.18 & 0.31 & 0.15 & 2.95 & 4.08 & 0.06 & 0.11 \\
\hline \multicolumn{9}{|c|}{ Salicylic acid } \\
\hline Control & 34.60 & 37.17 & 25.78 & 76.46 & 639.58 & 270.28 & 39.2 & 87.1 \\
\hline $100 \mathrm{ppm}$ & 38.66 & 37.93 & 27.19 & 81.41 & 700.14 & 319.17 & 50.9 & 105.1 \\
\hline $150 \mathrm{ppm}$ & 40.31 & 36.69 & 29.06 & 82.63 & 756.06 & 407.19 & 75.0 & 143.8 \\
\hline 200ppm & 41.52 & 37.72 & 29.81 & 85.16 & 811.50 & 473.61 & 87.3 & 174.6 \\
\hline LSD5\% & 0.19 & 0.12 & 0.23 & 0.21 & 5.11 & 3.18 & 0.09 & 0.11 \\
\hline \multicolumn{9}{|c|}{ Biofertilization } \\
\hline $\begin{array}{l}\text { Control } \\
(\mathrm{Br})\end{array}$ & 34.58 & 36.52 & 25.75 & 76.64 & 621.89 & 307.00 & 40.9 & 93.4 \\
\hline PDB & 37.60 & 38.05 & 27.56 & 79.98 & 708.67 & 355.64 & 59.7 & 120.9 \\
\hline PS & 40.29 & 39.32 & 28.67 & 83.16 & 745.25 & 382.50 & 71.6 & 136.2 \\
\hline $\begin{array}{l}\operatorname{mix} \\
(\mathrm{PDB}+ \\
\mathrm{BS})\end{array}$ & 42.62 & 40.62 & 29.86 & 85.87 & 831.47 & 425.11 & 87.4 & 158.6 \\
\hline LSD5\% & 0.23 & 0.15 & 0.26 & 0.21 & 4.72 & 3.48 & 0.06 & 0.14 \\
\hline \multicolumn{9}{|c|}{2011 Season } \\
\hline \multicolumn{9}{|c|}{ Sowing date } \\
\hline $20^{t h}$ Abril & 44.05 & 40.96 & 29.73 & 89.39 & 881.38 & 471.58 & 96.6 & 199.4 \\
\hline $15^{\text {th }}$ May & 39.31 & 39.46 & 28.31 & 86.86 & 810.19 & 435.31 & 70.6 & 155.1 \\
\hline $10^{\text {th }}$ June & 35.72 & 37.62 & 26.75 & 69.75 & 580.67 & 262.81 & 41.5 & 77.1 \\
\hline LSD5\% & 0.23 & 0.14 & 0.28 & 0.44 & 18.17 & 2.86 & 0.18 & 0.22 \\
\hline \multicolumn{9}{|c|}{ Salicylic acid } \\
\hline Control & 35.28 & 37.95 & 26.47 & 76.89 & 678.03 & 318.69 & 46.5 & 104.7 \\
\hline $100 \mathrm{ppm}$ & 39.45 & 38.95 & 27.61 & 81.76 & 726.64 & 331.83 & 53.5 & 111.6 \\
\hline $150 \mathrm{ppm}$ & 41.58 & 40.26 & 29.17 & 83.53 & 787.06 & 427.39 & 79.2 & 153.7 \\
\hline $200 \mathrm{ppm}$ & 42.472 & 40.29 & 29.81 & 85.83 & 837.92 & 481.69 & 99.9 & 181.5 \\
\hline LSD5\% & 0.42 & 0.17 & 0.27 & 0.36 & 10.08 & 4.26 & 0.14 & 0.12 \\
\hline \multicolumn{9}{|c|}{ Biofertilization } \\
\hline Control (Br) & 35.20 & 36.71 & 25.94 & 77.00 & 650.22 & 324.05 & 43.4 & 99.4 \\
\hline PDB & 38.39 & 38.79 & 28.00 & 80.38 & 749.47 & 380.11 & 64.7 & 133.2 \\
\hline PS & 41.45 & 40.32 & 28.86 & 83.79 & 772.58 & 405.97 & 76.4 & 147.6 \\
\hline $\begin{array}{l}\operatorname{mix}(\mathrm{PDB} \\
+\mathrm{BS})\end{array}$ & 43.73 & 41.57 & 30.25 & 86.83 & 857.36 & 449.47 & 93.4 & 171.1 \\
\hline LSD5\% & 0.38 & 0.20 & 0.25 & 0.45 & 5.03 & 3.76 & 0.12 & 0.18 \\
\hline
\end{tabular}

Egypt. J. Agron. 35, No. 1 (2013) 
The maximum value of plant height $(42.62$ and $43.73 \mathrm{~cm})$, weight of pods per plant( 10.62 and $11.57 \mathrm{~g}$ ), number of pods per plant (9.86 and 10.25), 100 seeds weight ( 35.87 and $36.83 \mathrm{~g}$ ), pod yields $(831.47$ and $857.36 \mathrm{~kg} / \mathrm{fad}$ ) and seed yields (425.11 and $449.47 \mathrm{~kg} / \mathrm{fad}$ ) protein combined yield ( 87.4 and 93.4 $\mathrm{kg} / \mathrm{fad}$ ) and oil yields (158.6 and $171.1 \mathrm{~kg} / \mathrm{fad})$ were obtained by biofertilized peanut plant with mixed biofertilization treatments $(\mathrm{PDB}+\mathrm{PS})$ through the first and second seasons, respectively. These results in accordance with Badawi et al. (2011), they reported that Co inoculation of Peanut with Bradyrhizobium and PGPRs significantly magnified the Peanut yield (41\%) during two growing seasons, synergy inoculation between Bradyrhizobium and PGPRs led to further increases in all studied parameters and strengthened the stimulating effect of bacterial inoculation.

Regarding the effect of interaction between sowing date and biofertilization (Table 6), on plant height, weight of pods per plant, number of pods per plant, 100-seeds weight, pod and seed yields, protein and oil yields of peanut through 2010 and 2011seasons, data indicated significant increase of the previous mentioned parameters.

Above mentioned results may be due to the suitable temperature degrees which led to increase seeds yield from biofertilzed plant seed that sown on April $20^{\text {th }}$ with mix two type of bacteria at the period from flowering to harvest, this caused an increase in dry matter accumulation in plant organs which reflected on plant height, weight of pods per plant number of pods per plant, 100 seed weight.

The interaction effect of sowing date and salicylic acid foliar application reached the 5\% level of significance for the yield of peanut and its attributes; i.e., plant height $(\mathrm{cm})$, weight of pods per plant $(\mathrm{g})$, number of pods per plant, 100 seeds weight $(\mathrm{g})$, pod and seed yields ( $\mathrm{kg} / \mathrm{fad})$ and protein and oil yields $(\mathrm{kg} / \mathrm{fad})$, in both seasons (Table 7). It could be conclude that there was an opposite relation between delaying date of sowing peanut seeds and increasing of salicylic acid foliar application on the yield of peanut and its components characteristics through 2010 and 2011. It could be concluded that increasing rate of salicylic acid led to productive seeds than vegetative growth. However, the maximum value of seed yields protein and oil yields (kg/fad) (575.6, 132.9 and 251.5 in the 2010 and 598.1, 138.8 and 263.8 in 2011) respectively, were obtained from plants grown in the $20^{\text {th }}$ of April treated with $150 \mathrm{ppm}$ salicylic acid. While in the second date May $15^{\text {th }}$ treated plant with 200 ppm salicylic acid. This is to be logic since the same interaction gained the highest values of yield components and consequently seed yield (kg/fad) (Metwally et al., 2003). 
TABLE 6 . Effect of interaction between sowing dates and biofetilization on yield and its components of peanut in the two growing seasons.

\begin{tabular}{|c|c|c|c|c|c|c|c|c|}
\hline Characters & $\begin{array}{c}\text { Plant } \\
\text { height } \\
\text { (cm) }\end{array}$ & $\begin{array}{l}\begin{array}{c}\text { Weight } \\
\text { of } \\
\text { pods/ } \\
\text { plants } \\
\text { (g) }\end{array} \\
\end{array}$ & $\begin{array}{c}\text { No. } \\
\text { of } \\
\text { pods/ } \\
\text { plants }\end{array}$ & $\begin{array}{c}\text { 100- } \\
\text { seeds } \\
\text { weight } \\
\text { (g) }\end{array}$ & $\begin{array}{c}\text { Pod } \\
\text { yield } \\
\text { (kg/fad) }\end{array}$ & $\begin{array}{c}\text { Seed } \\
\text { yield } \\
\text { (kg/fad) }\end{array}$ & $\begin{array}{c}\text { Protein } \\
\text { yield } \\
\text { (kg/fad) }\end{array}$ & $\begin{array}{l}\text { Oil yield } \\
\text { (kg/fad) }\end{array}$ \\
\hline \multicolumn{9}{|c|}{2010 Season } \\
\hline D1 B0 & 38.4 & 7.7 & 6.4 & r., & $\varkappa 1, v$ & $r+v, \varepsilon$ & 50.3 & 123.1 \\
\hline B1 & $\varepsilon 1, v$ & 9,0 & $\wedge, \vee$ & rᄉ, & Nrr, & $\varepsilon r r, \wedge$ & 86.1 & 173.6 \\
\hline $\mathrm{B} 2$ & $\varepsilon 0$, & $1 \cdot, v$ & $1 \cdot, \cdot$ & $\varepsilon \cdot, \uparrow$ & Nדr,r & $\{71,1$ & 104.2 & 201.0 \\
\hline B3 & $\varepsilon \Lambda, r$ & Ir, r & 11,0 & $\varepsilon r, \varepsilon$ & $1 \cdots 9, r$ & Or., \& & 129.6 & 231.0 \\
\hline D2 $\mathrm{B} 0$ & $r r, r$ & $7, r$ & $7, \cdot$ & rT, \& & $700, r$ & rtr, & 42.9 & 97.5 \\
\hline B1 & $r v, r$ & $\wedge, \cdot$ & $\vee, \wedge$ & $r \varepsilon, 1$ & vol,r & rᄉr,r & 59.3 & 127.7 \\
\hline $\mathrm{B} 2$ & $\varepsilon \cdot, \cdot$ & $q, r$ & $\wedge, q$ & $r v, \wedge$ & $\vee 99, v$ & $\varepsilon r 1, V$ & 71.7 & 150.5 \\
\hline B3 & $\varepsilon r, r$ & 11, & $1 \cdot, r$ & $\varepsilon \cdot, v$ & $\wedge \wedge \uparrow, \vee$ & $\varepsilon \vee V, 0$ & 88.8 & 178.1 \\
\hline D3 $\quad$ B0 & rr, & 0,7 & $\varepsilon, q$ & $1 \varepsilon, 9$ & 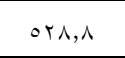 & $r r \cdot, t$ & 30.2 & 63.6 \\
\hline $\mathrm{B} 1$ & $r r, q$ & $7, V$ & $7, r$ & $1 \mathrm{~V}, \wedge$ & $001, \mathrm{~V}$ & $r 01,9$ & 37.5 & 72.0 \\
\hline B2 & $r 0, q$ & $v, q$ & $\mathrm{v}, 1$ & $r, r$ & 572.8 & $r T \leqslant, \Lambda$ & 43.7 & 78.1 \\
\hline B3 & $r v, \varepsilon$ & $\Lambda, \vee$ & $\mathrm{v}, \mathrm{V}$ & $r r, t$ & $090, r$ & $r V V, \varepsilon$ & 50.2 & 84.1 \\
\hline $\begin{array}{l}\text { LSD at } \\
5 \%\end{array}$ & $\cdot, 9$ & $\cdot, 7$ & 1,1 & $\cdot, 9$ & $r \cdot, l$ & $1 \leqslant, \wedge$ & $\cdot, r$ & $\cdot, 7$ \\
\hline \multicolumn{9}{|c|}{2011 Season } \\
\hline D1 B0 & rᄉ,, & $v, q$ & 7,9 & rr,r & $V \backslash \leq, 0$ & $r q \varepsilon, \varepsilon$ & 54.4 & 132.5 \\
\hline $\mathrm{B} 1$ & $\varepsilon r, T$ & $1 \cdot, r$ & $9, v$ & rᄉ, $\varepsilon$ & $\wedge \vee 7, \cdot$ & $\varepsilon 07,9$ & 91.8 & 184.6 \\
\hline $\mathrm{B} 2$ & 45.5 & 11,9 & $1 \cdot, r$ & $\varepsilon 1, r$ & А१r,v & $\varepsilon \wedge \varepsilon, \wedge$ & 115.0 & 203.1 \\
\hline B3 & $\varepsilon q, r$ & $1 \pi, 7$ & $1 r, 1$ & $\varepsilon \varepsilon, V$ & $1 \cdot r 0,7$ & $00 \cdot r$ & 139.2 & 246.5 \\
\hline D2 $\quad$ B0 & $r r, v$ & 7,0 & 0,9 & rT, T & $7 \vee 9,$. & $r \varepsilon \cdot, \cdot$ & 45.6 & 104.4 \\
\hline B1 & $r v, q$ & 9,1 & $v, q$ & $r \varepsilon, q$ & Vqv, I & $\varepsilon r \varepsilon,$. & 66.6 & 152.2 \\
\hline $\mathrm{B} 2$ & $\sum 1,9$ & $1 \cdot, 0$ & $9, \cdot$ & rᄉ, 7 & Nrr,o & $\varepsilon \pi r, 0$ & 79.1 & 172.1 \\
\hline B3 & $\varepsilon r, r$ & 11,1 & $1 \cdot, 0$ & $\sum 1,7$ & $94, r$ & $01 \varepsilon, 1$ & 96.3 & 198.7 \\
\hline D3 BO & rt,o & $7, \cdot$ & 0,1 & $10, r$ & $0 \leq \Lambda$, & $r r v, v$ & 31.6 & 65.8 \\
\hline $\mathrm{B} 1$ & $r \varepsilon, 7$ & $\mathrm{v}, 1$ & $7, \varepsilon$ & 11,1 & ovo,r & $r \circ q, v$ & 39.7 & 74.8 \\
\hline $\mathrm{B} 2$ & $r v,$. & $\wedge, \varepsilon$ & $v, r$ & rI, o & $09 \cdot, 7$ & $r V \cdot, V$ & 44.9 & 81.2 \\
\hline B3 & $r \wedge, \wedge$ & $9, r$ & $\wedge, 1$ & $r \varepsilon, r$ & $7 \cdot \wedge, \wedge$ & r人r, & 51.9 & 87.3 \\
\hline $\begin{array}{l}\text { LSD at } \\
5 \%\end{array}$ & 1,7 & $\cdot, 9$ & 1,1 & 1,9 & $r 1, \varepsilon$ & 17 & $\cdot, 0$ & $\cdot, \wedge$ \\
\hline
\end{tabular}

$\mathrm{B} 0=$ Bradyrhizobium, $($ control $), \mathrm{B} 1=$ B. megatherium var. phosphaticum $(\mathrm{PDB}), \mathrm{B} 2=$ Pseudomonas fluorescens (PS), and B3 $=$ mix between $(\mathrm{PDB}+\mathrm{PS})$ while, D1 $=20^{\text {th }}$ April, D2 $=15^{\text {th }}$ May and D3 $=10^{\text {th }}$ June

Egypt. J. Agron. 35, No. 1 (2013) 
EFFECT OF SALICYLIC ACID, BIOFERTILIZATION ...

TABLE 7 . Effect of interaction between sowing date and salicylic acid on yield and its components of peanut in the two growing seasons .

\begin{tabular}{|c|c|c|c|c|c|c|c|c|}
\hline Characters & $\begin{array}{c}\text { Plant } \\
\text { height } \\
(\mathrm{cm})\end{array}$ & $\begin{array}{c}\text { Weigh } \\
\text { of } \\
\text { pods }\end{array}$ & $\begin{array}{c}\begin{array}{c}\text { No. } \\
\text { of } \\
\text { pods/ }\end{array} \\
\text { plants }\end{array}$ & $\begin{array}{c}\text { 100- } \\
\text { seeds } \\
\text { weight } \\
\text { (g) }\end{array}$ & $\begin{array}{c}\text { Pod } \\
\text { yield } \\
(\mathrm{kg} / \mathrm{fad}\end{array}$ & $\begin{array}{c}\text { Seed } \\
\text { yield } \\
\text { (kg/fad) }\end{array}$ & $\begin{array}{c}\text { Protein } \\
\text { yield } \\
\text { (kg/fad) }\end{array}$ & $\begin{array}{l}\text { Oil yield } \\
\text { (kg/fad) }\end{array}$ \\
\hline & \multicolumn{8}{|c|}{2010 season } \\
\hline D1 SA0 & 40.64 & $v, v r$ & $7, r_{0}$ & $r_{0,90}$ & $V \leqslant r, r_{O}$ & 314.40 & 53.7 & 116.6 \\
\hline SA1 & 42.35 & $q, r$. & $\Lambda, r_{0}$ & ra,rr & $\lambda \cdot r_{\text {, }}$ & 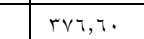 & 73.8 & 143.5 \\
\hline SA2 & 45.31 & Ir,r. & ${ }_{0}^{11, r}$ & $\leqslant 1, \cdots$ & 929.15 & ovo,r. & 132.9 & 251.5 \\
\hline SA3 & $\varepsilon 0, \cdot \wedge$ & $1 \cdot, \wedge$ & $1 \cdot, 0$. & rᄉ, r. & $q \cdot r, \cdot \wedge$ & $010, \cdot 1$ & 109.7 & 210.2 \\
\hline D2 $\mathrm{SA} 0$ & $r \varepsilon, r r$ & $v, 10$ & 6.25 & $r+10$ & $797, r r$ & TVT,\&T & 36.6 & 86.4 \\
\hline SA1 & rv, v八 & $\vee, 90$ & $V, r_{0}$ & $r_{0,10}$ & $V Y \Lambda, \wedge 0$ & $r_{0}, r_{0}$ & 47.3 & 112.5 \\
\hline SA2 & $r q, r$. & $9, r$. & 8.55 & $r_{0, \Lambda \Lambda}$ & $V 7 \varepsilon, 9 T$ & $\varepsilon \cdot \varepsilon, r_{0}$ & 64.3 & 133.8 \\
\hline SA3 & $\leqslant 1, Y \wedge$ & $1 \cdot, r$. & $11, \cdots$ & $\leq 1, \cdot r$ & Qrr, ४ & $\Delta V \checkmark, \varepsilon r$ & 120.5 & 228.8 \\
\hline D3 SA0 & $r \wedge, \wedge \Lambda$ & $7,7 \wedge$ & $\varepsilon, v_{0}$ & $11, r_{0}$ & $01 \cdot, 11$ & rrr,,. & 28.7 & 62.4 \\
\hline SA1 & $r_{0,10}$ & $\uparrow, \wedge$ & $7,1$. & $19, \cdot r$ & $07 V, \wedge r$ & $r r \cdot, 01$ & 32.5 & 66.2 \\
\hline SA2 & ru, to & $v, \varepsilon 0$ & $\vee, 1 \wedge$ & $r \cdot, 9 \wedge$ & $\circ \vee \varepsilon, \cdot \Lambda$ & $r \leqslant 1, V_{0}$ & 39.2 & 70.6 \\
\hline SA3 & $r v, q$ & 1,10 & $\vee, \wedge r$ & $r \uparrow, r$. & $097, r r$ & rrq,rr & 64.2 & 99.1 \\
\hline LSD at 5\% & $\cdot, V V I r$ & 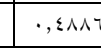 &., $91 \% 1$ & ., ২ঙq & r., Trר & $1 \mathrm{r}, 1011$ & 0.3604 & 0.1771 \\
\hline & & & & 01 ) seaso & & & & \\
\hline D1 $\quad$ SA0 & 41.15 & $\wedge, \wedge r$ & V,rr & $r q, \varepsilon 0$ & Vos,,. & $r_{T \Lambda}, v_{0}$ & 63.8 & 137.2 \\
\hline SA1 & 42.74 & $1 \cdot, \leqslant 0$ & $\Lambda, v_{0}$ & rq, v. & $\Delta r \varepsilon, 1 \wedge$ & 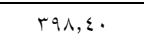 & 79.7 & 152.2 \\
\hline SA2 & $\leq 4.2$ & 19,90 & $11, \wedge 0$ & $\leq r, 1$. & $9 \wedge \bullet, \wedge \bullet$ & 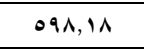 & 138.8 & 263.8 \\
\hline SA3 & $\varepsilon 7,1 r$ & $11,0$. & $11, \cdot 1$ & $r q, r \wedge$ & $9 \leqslant 0,11$ & or,$\ldots$ & 112.0 & 214.7 \\
\hline $\begin{array}{ll}\text { D2 } & \text { SA0 }\end{array}$ & $r \leqslant, v_{0}$ & $\Lambda, \cdot r$ & 7,70 & rr, Th & VIV, 10 & $r o \varepsilon, .$. & 47.8 & 116.8 \\
\hline SA1 & $r \wedge, \wedge \wedge$ & $9, \cdot 1$ & $\vee, \uparrow \wedge$ & $r q, r$. & V74, ^० & ror,qr & 47.8 & 119.9 \\
\hline SA2 & $\varepsilon \cdot, \wedge r$ & $1 \cdot, \cdot$. & ᄉ, rr & $r q, q r$ & จง, 9. & $\varepsilon r v, r_{0}$ & 69.9 & 149.9 \\
\hline SA3 & 42.95 & $1 \cdot, \mathrm{V} \wedge$ & $1 \cdot, 7 \wedge$ & $\leqslant 1, \leqslant \wedge$ & 979,10 & $\bullet \wedge \wedge, \diamond \wedge$ & 124.8 & 243.1 \\
\hline $\begin{array}{ll}\text { D3 } & \text { SA0 }\end{array}$ & rq,qr & $7, \wedge 0$ & 0,0 r & $11, \varepsilon r$ & oor,o. & Trt,ro & 30.6 & 66.3 \\
\hline SA1 & ru, vo & $V, r_{0}$ & $7,0$. & $19, \mathrm{r \Lambda}$ & ov^,qr & rro, ro & 33.5 & 68.1 \\
\hline SA2 & $r_{v, q}$. & $\wedge, 1$. & $v, r_{0}$ & ri,or & $0 \wedge \Lambda, \varepsilon r$ & $r \leqslant \uparrow, 9 \wedge$ & 40.5 & 73.1 \\
\hline SA3 & rᄉ, ro & ᄉ,or & $v, 70$ & ru, vo & T.r,Ar & rro,or & 65.7 & 102.3 \\
\hline $\begin{array}{c}\text { LSD at } \\
5 \%\end{array}$ & 1.2952 & $\cdot, 7 \wedge \wedge 0$ & $1,1.99$ & $1, \varepsilon V \leqslant V$ & $\varepsilon \cdot, V T \cdot V$ & 18,1911 & 0.5737 & 0.4941 \\
\hline
\end{tabular}

$\mathrm{D} 1=20^{\text {th }}$ April, D2 $=15^{\text {th }}$ May and D3 $=10^{\text {th }}$ June, While SA0 $=$ zero salicylic acid, SA1 $=100 \mathrm{ppm}$, $\mathrm{SA} 2=150 \mathrm{ppm}$ and $\mathrm{SA} 3=200 \mathrm{ppm}$

For the interaction effect between salicylic acid and biofertilization (Fig. 1a -1g) data shown significant effects on plant height, weight of pods per plant, number of pods per plant, 100-seed weight, pod and seed yields, protein and oil yields in both seasons. The obtained data showed that increasing salicylic acid rates from 
100 to $200 \mathrm{ppm}$ and biofertlized with mixture of Bacillus megatherium and Pseudomonas fluorescence (the two types of bacteria) in both seasons caused an increase in all aforementioned parameters. It may be due to that increasing both salicylic acid rates and mix biofertiliztion together led to an encouragement in seed formation owing to increasing the plant capacity in building metabolites and caused increases in seed and pod yields.

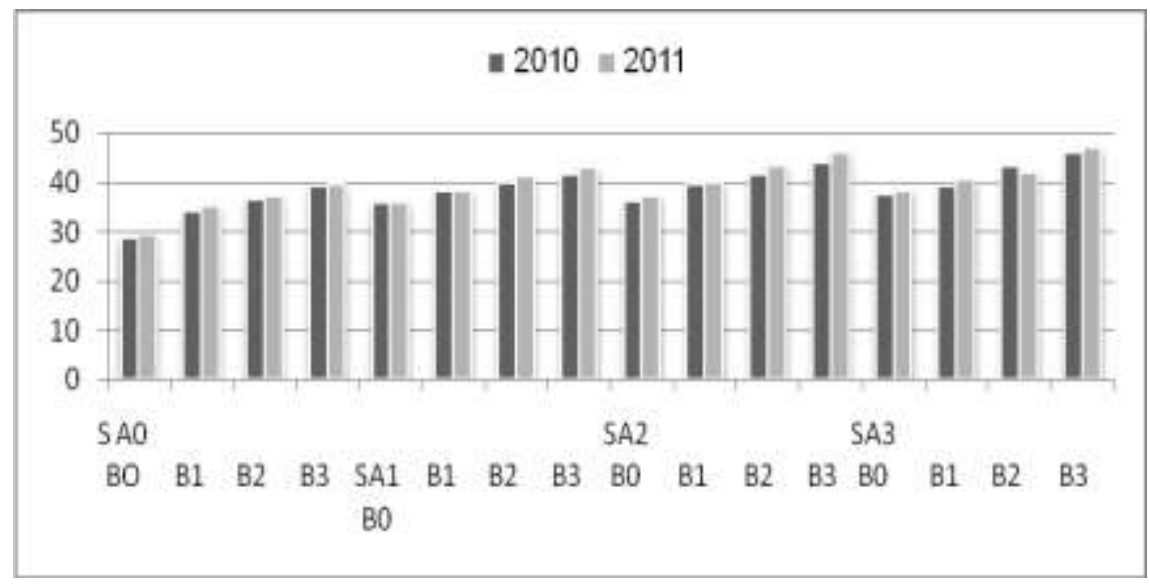

Fig - 1a. Effect of interaction between salicylic acid and biofertlization on plant height $(\mathbf{c m})$ of peanut in two growing seasons .

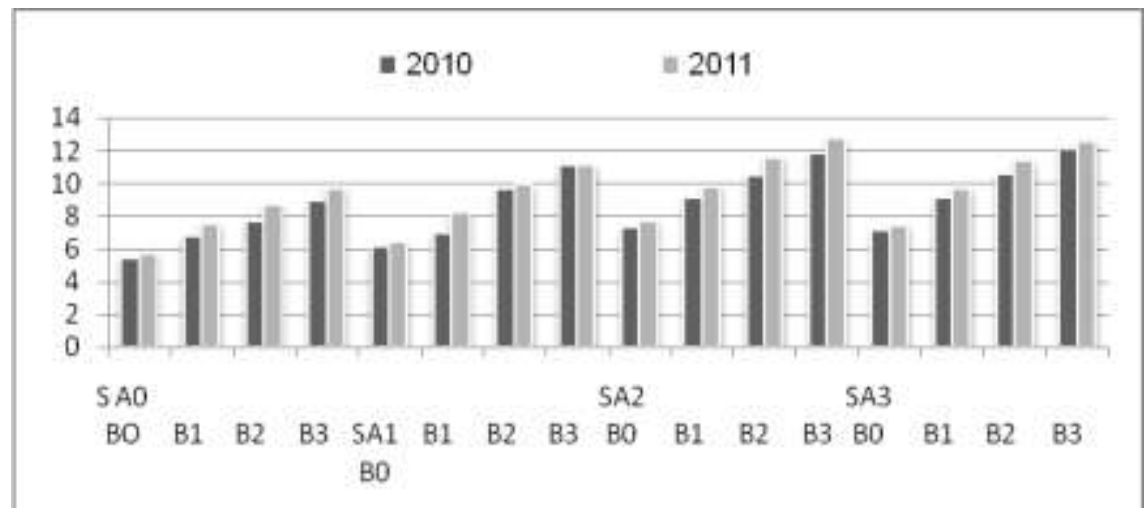

Fig . 1b. Effect of interaction between salicylic acid and biofertlization on weight of pod (g) of peanut in two growing seasons.

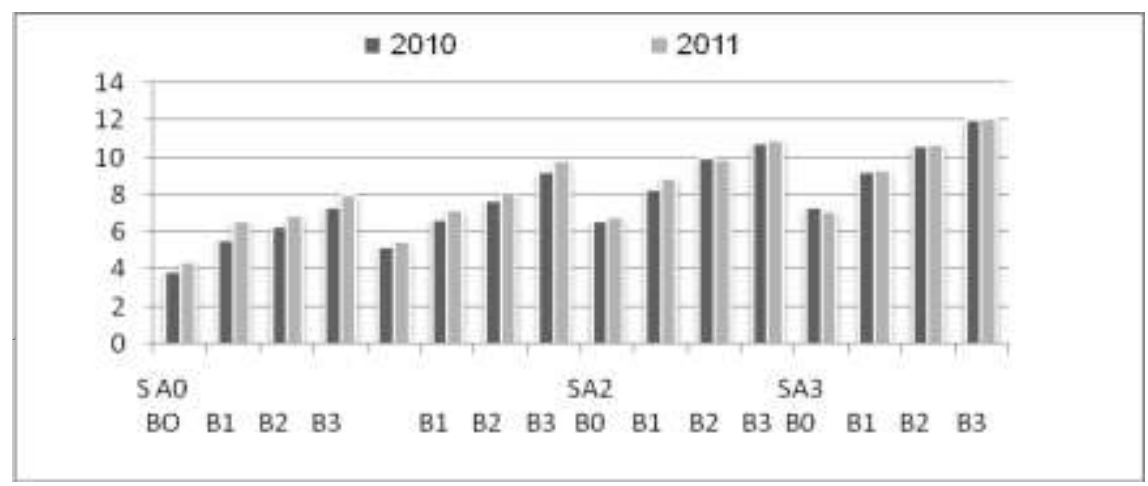


Fig . 1c. Effect of interaction between salicylic acid and biofertlization on No. of pod/plant of peanut in two growing seasons .

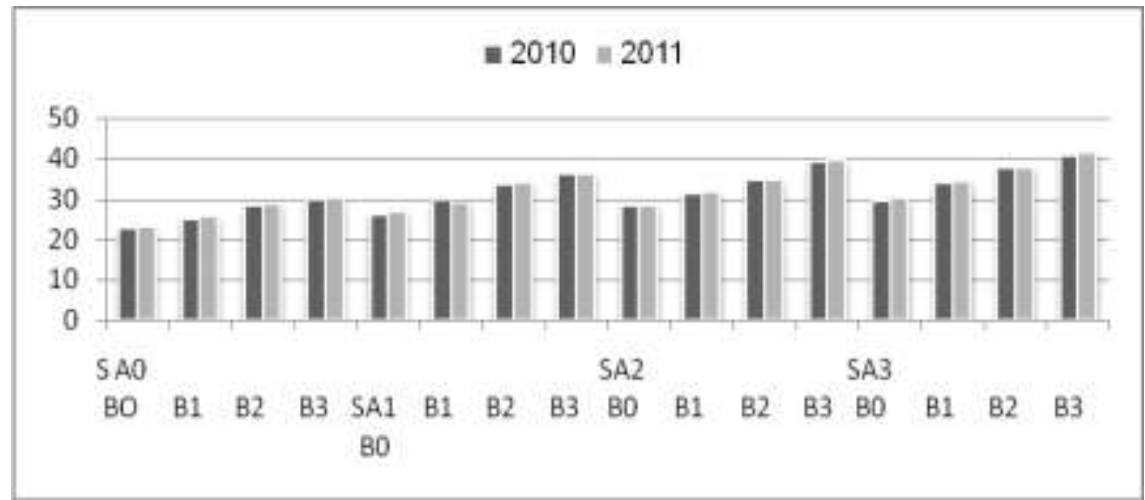

Fig . 1d. Effect of interaction between salicylic acid and biofertlization on weight of 100 seed $(\mathrm{g})$ of peanut in two growing seasons .

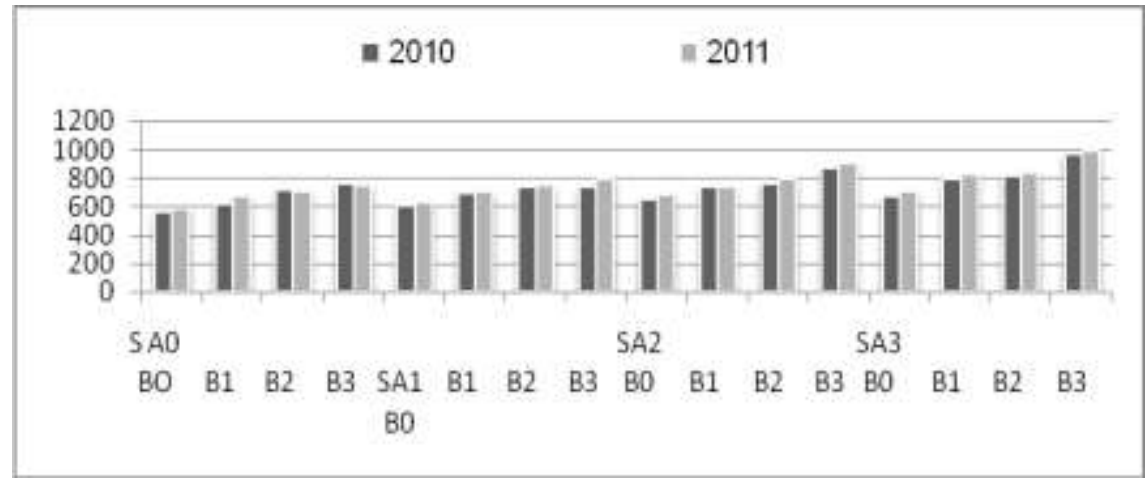

Fig .1e. Effect of interaction between salicylic acid and biofertlization on pod yield $(\mathrm{kg} / \mathrm{fad})$ of peanut in two growing seasons .

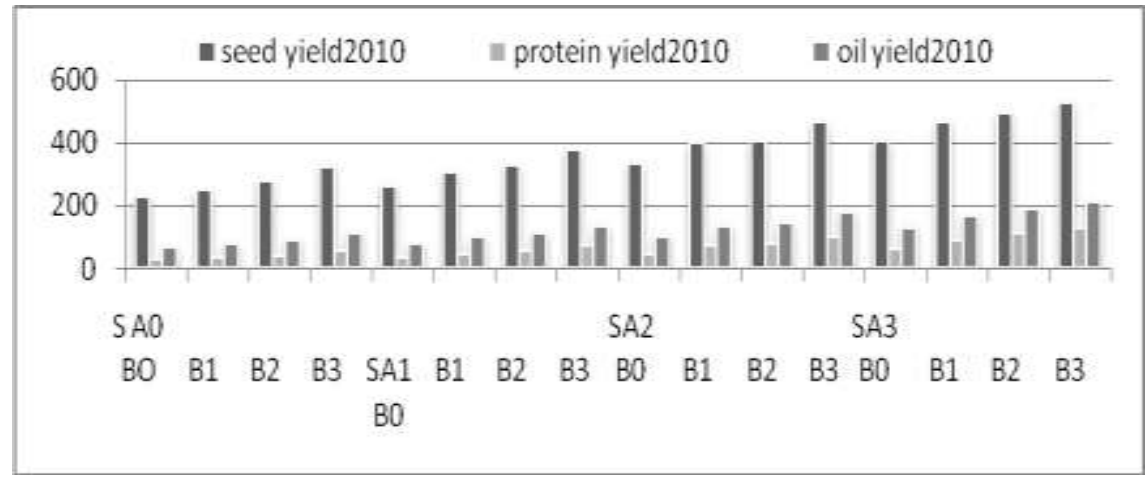

Egypt. J. Agron. 35, No. 1 (2013) 
Fig - 1f. Effect of interaction between salicylic acid and biofertlization on seed, protein and oil yields $(\mathrm{kg} / \mathrm{fad})$ of peanut in 2010 season.

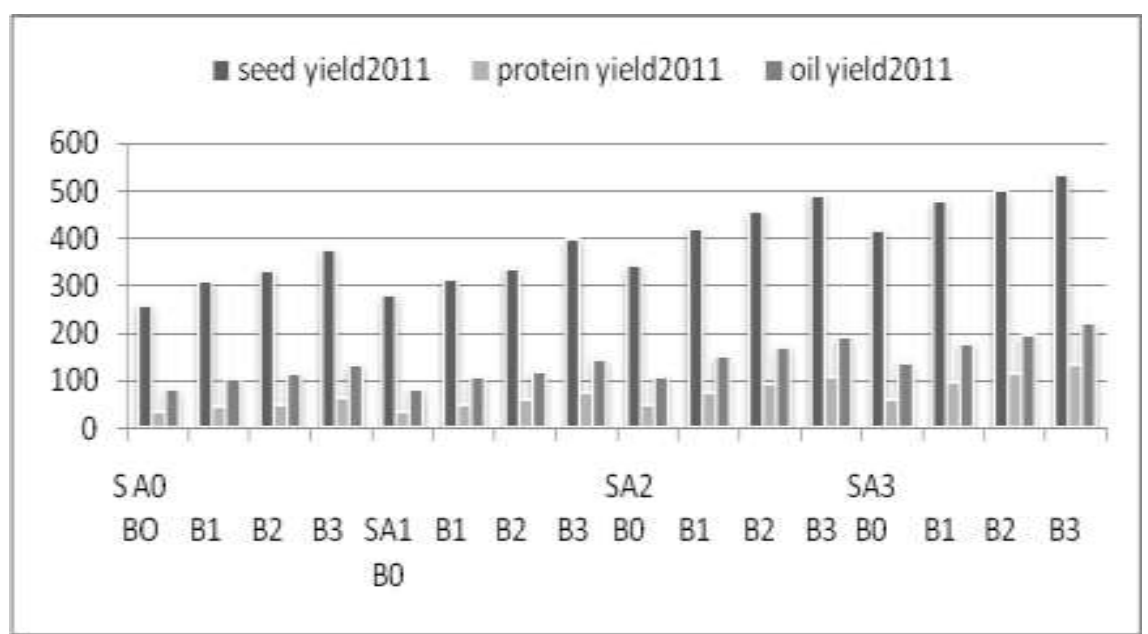

Fig - 1g. Effect of interaction between salicylic acid and biofertlization on seed, protein and oil yields of peanut in 2011 season.

$\mathrm{SA} 0=$ zero salicylic acid, $\mathrm{SA} 1=100 \mathrm{ppm}, \mathrm{SA} 2=150 \mathrm{ppm}$ and $\mathrm{SA} 3=200 \mathrm{ppm}$ While $\mathrm{B} 0=$ Bradyrhizobium, (control), B1 = B. megatherium var. phosphaticum $(\mathrm{PDB}), \mathrm{B} 2=$ Pseudomonas fluorescens $(\mathrm{PS})$, and $\mathrm{B} 3=$ mix between $(\mathrm{PDB}+\mathrm{PS})$

Applied biofertilization enhanced the utilization of applied salicylic acid could play a major role during the early stages of Bradyrhizobium-legume symbiosis. Nodulation factors produced by rhizobia, in response to legume produced flavonoids, affected SA content of the host plant during the early stages of nodulation. The maximum values of plant height, weight of pods per plant number of pod per plant, 100-seed weight, pod and seed yields, and protein and oil yields were obtained from plants sown on April $20^{\text {th }}$ and supplied with $200 \mathrm{ppm}$ salicylic acid and biofertilized with mix of two types of bacteria. This was true for the studied two seasons. Generally, the addition of biofertilizer with salicylic acid led to improvement the quantity and quality yield chacterization as compared to salicylic acid alone (Bai et al., 2002).

The effect of interaction between salicylic acid foliar application, biofertilization and sowing dates was significant for yield and its components characteristics of peanut in both seasons (Tables $8 \mathrm{a}$ and $8 \mathrm{~b}$ ). The maximum value of plant height, weight of pods per plant number of pod per plant, 100-seed weight, pod and seed yields and protein and oil yields were obtained at growing seasons 2010 and 2011, from plants sown on April $20^{\text {th }}$ and supplied with $150 \mathrm{ppm}$ salicylic acid and mixed biofertilization treatments . 
TABLE 8a. Effect of interaction between salicylic acid and biofertilization and sowing date on yield and its components of peanut in 2010 season .

\begin{tabular}{|c|c|c|c|c|c|c|c|c|c|}
\hline \multicolumn{2}{|c|}{ Characters } & $\begin{array}{c}\text { Plant } \\
\text { height } \\
(\mathrm{cm})\end{array}$ & $\begin{array}{l}\text { Weight } \\
\text { of } \\
\text { pods/ } \\
\text { plants } \\
\text { (g) }\end{array}$ & $\begin{array}{c}\text { No. } \\
\text { of pods/ } \\
\text { plants }\end{array}$ & $\begin{array}{c}100- \\
\text { seeds } \\
\text { weight } \\
\text { (g) }\end{array}$ & $\begin{array}{c}\text { Pod } \\
\text { yield } \\
\text { (kg/fad }\end{array}$ & $\begin{array}{c}\text { Seed } \\
\text { yield } \\
(\mathrm{kg} / \mathrm{fad})\end{array}$ & $\begin{array}{c}\text { Protein } \\
\text { yield } \\
(\mathbf{k g} / \mathrm{fad})\end{array}$ & $\begin{array}{l}\text { Oil yield } \\
\text { (kg/fad) }\end{array}$ \\
\hline \multirow[t]{4}{*}{ D1 SA0 } & B0 & $r_{4}, \varepsilon$ & 0,7 & $\varepsilon, V$ & $r \cdot, \varepsilon$ & $71 \cdot, r$ & $r \leqslant 7, r$ & 31.3 & 78.6 \\
\hline & $\mathrm{B} 1$ & ґ৯, , & $V, r$ & $0, \mathrm{~V}$ & rr,o & $V \cdot Y, V$ & 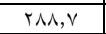 & 47.3 & 107.9 \\
\hline & $\mathrm{B} 2$ & $\varepsilon 1, \wedge$ & $\Lambda, 0$ & $7, V$ & $r q, r$ & v^r, $\vee$ & $r Y Y, r$ & 59.7 & 125.9 \\
\hline & B3 & $\varepsilon 0, V$ & 9,0 & $\Lambda, r$ & $\varepsilon \cdot, V$ & $\Lambda \vee Y, r$ & $r 99, r$ & 82.8 & 160.5 \\
\hline \multirow[t]{4}{*}{ SA1 } & B0 & $r_{\Lambda, 0}$ & $v, 1$ & $0, \mathrm{~V}$ & $r Y, T$ & $707, r$ & rی१,. & 37.6 & 94.5 \\
\hline & $\mathrm{B} 1$ & $\varepsilon \cdot, \varepsilon$ & $\Lambda, r$ & $v, r$ & $r 9,0$ & Arr,. & $\mathrm{r} 7 \mathrm{Y}, \mathrm{V}$ & 70.4 & 141.5 \\
\hline & $\mathrm{B} 2$ & $\varepsilon r, \wedge$ & $9, \wedge$ & $\Lambda, \vee$ & $\varepsilon 1, v$ & $10 \cdot, \cdot$ & $r v q, v$ & 84.3 & 149.2 \\
\hline & B3 & $\sum 7, V$ & $11, \mathrm{~V}$ & $11, r$ & $\varepsilon r, 0$ & $\Lambda \Lambda 0, v$ & $\varepsilon v 1, \cdot$ & 112.6 & 195.9 \\
\hline \multirow[t]{4}{*}{ SA2 } & B0 & rᄉ,r & 9,7 & $\mathrm{v}, \mathrm{v}$ & ry,. & $V \leqslant r,$. & $\varepsilon \wedge \cdot\rangle$, & 70.7 & 172.6 \\
\hline & B1 & $\varepsilon \varepsilon, 7$ & 11,9 & $11, r$ & $\varepsilon \cdot r$ & $\Lambda \wedge r, \cdot$ & ovi, V & 129.2 & 248.1 \\
\hline & $\mathrm{B} 2$ & $\varepsilon 7, V$ & $1 Y, \Lambda$ & $\mathrm{Ir}, \mathrm{V}$ & $\varepsilon 1, r$ & $q 1 r, r$ & $T \cdot \varepsilon,$. & 159.5 & 274.2 \\
\hline & B3 & $0 ., \mathrm{V}$ & $1 \varepsilon, 9$ & $1 \%, V$ & $\varepsilon 7, \varepsilon$ & $\Pi \vee V \wedge, r$ & $7 \leqslant 7, \cdot$ & 185.4 & 323.0 \\
\hline \multirow[t]{4}{*}{ SA3 } & B0 & $\varepsilon \cdot, 0$ & $\Lambda, r$ & $v, r$ & $r, r$ & $\vee \wedge, \cdot$ & Eor, $v$ & 65.3 & 151.9 \\
\hline & B1 & $\varepsilon r, q$ & $1 \cdot, \varepsilon$ & $1 \cdot, r$ & $r \wedge, v$ & $\Lambda \Lambda r, v$ & $0 . \varepsilon,$. & 107.8 & 206.1 \\
\hline & B2 & $\sum \vee, \wedge$ & $11, \mathrm{~V}$ & $11, V$ & $\varepsilon \cdot, \cdot$ & $9 \cdot 7, r$ & or $\varepsilon, r$ & 124.5 & 232.4 \\
\hline & B3 & $0 .,$. & $1 Y, \Lambda$ & Ir,V & $\varepsilon r, \wedge$ & $11 \cdots, r$ & $04 \Lambda, r$ & 148.9 & 258.0 \\
\hline \multirow[t]{4}{*}{ D2 SA0 } & B0 & $r_{0, q}$ & 0,0 & $\varepsilon, \cdot$ & $r V, V$ & $r \cdot r, \cdot$ & $r M, r$ & 28.4 & 65.4 \\
\hline & $\mathrm{B} 1$ & $r \varepsilon, v$ & 7,7 & $7, r$ & $r \cdot, r$ & $7 \varepsilon \wedge, r$ & $r \leqslant \leqslant, \cdot$ & 31.2 & 75.2 \\
\hline & $\mathrm{B} 2$ & $r v, r$ & $\mathrm{v}, \varepsilon$ & $\mathrm{v}, \mathrm{.}$ & $r r, q$ & $79 \cdot, v$ & $r \vee \wedge, V$ & 36.8 & 91.7 \\
\hline & B3 & $r q, \varepsilon$ & 9,1 & $\mathrm{~V}, \mathrm{~V}$ & $r 4, \varepsilon$ & $V Y \varepsilon, r$ & $r r q, v$ & 52.6 & 117.2 \\
\hline \multirow[t]{4}{*}{ SA1 } & B0 & $r o, r$ & 7,1 & $0, r$ & $r, \Lambda$ & $7 r v, v$ & $r \wedge 0, \cdot$ & 35.6 & 80.4 \\
\hline & B1 & $r V, r$ & $V, r$ & $\mathrm{v}$, & rr,. & $79 \mathrm{~V}, \mathrm{~V}$ & Trt,. & 44.7 & 101.4 \\
\hline & $\mathrm{B} 2$ & $r \wedge, \wedge$ & 1,0 & $\mathrm{~V}, \mathrm{~V}$ & $r v, v$ & $v 79, r$ & $r+\mathrm{r}, \mathrm{V}$ & 53.6 & 123.9 \\
\hline & B3 & $r q, 9$ & $1 \cdot, \cdot$ & $9,$. & $\varepsilon \cdot, q$ & $\Lambda 1 \cdot, V$ & $\varepsilon r \uparrow, V$ & 67.8 & 148.9 \\
\hline \multirow[t]{4}{*}{ SA2 } & B0 & ro,r & $7, \varepsilon$ & $7, r$ & rr, q & $777,$. & $r q \leqslant, V$ & 40.4 & 86.1 \\
\hline & $\mathrm{B} 1$ & rᄉ, $\varepsilon$ & $\Lambda, 0$ & $\Lambda, r$ & $r \varepsilon, r$ & V०ᄉ, & $r a r, r$ & 61.4 & 128.6 \\
\hline & $\mathrm{B} 2$ & $\varepsilon \cdot 0$ & $1 \cdot, \mathrm{V}$ & $9, r$ & $r 4, v$ & $\wedge \cdots, \cdot$ & $\sum r_{0},$. & 72.2 & 150.1 \\
\hline & B3 & $\varepsilon Y, T$ & 11,7 & $1 \cdot, r$ & $r q, v$ & Aro, $\mathrm{V}$ & $\varepsilon q\{, \cdot$ & 87.9 & 177.3 \\
\hline \multirow[t]{4}{*}{ SA3 } & B0 & $r+r$ & $\mathrm{v}, 1$ & $\Lambda, r$ & $r v, 1$ & $v 10,$. & $\{\wedge,$, & 70.2 & 167.9 \\
\hline & B1 & $r \wedge, r$ & $q, v$ & $q, v$ & $r \wedge, 7$ & $9 \cdot 1, \cdot$ & $079, \mathrm{~V}$ & 112.2 & 218.8 \\
\hline & $\mathrm{B} 2$ & $\varepsilon r, 0$ & $1 \cdot, \mathrm{V}$ & $11, V$ & $\varepsilon r, V$ & $9 \curlyvee \wedge, \vee$ & $7 \cdot 0, r$ & 145.3 & 251.2 \\
\hline & B3 & $\leqslant \square, V$ & $1 \mu, r$ & $1 \leqslant, r$ & $\varepsilon \theta, V$ & $11 \vee 4, \cdot$ & $\checkmark \leq 9, V$ & 164.4 & 285.2 \\
\hline \multirow[t]{4}{*}{ D3 SA0 } & B0 & $r r, 0$ & $0, \cdot$ & $r, \cdot$ & $1 \cdot, \varepsilon$ & $\varepsilon 7 \cdot, V$ & $r \cdot o, \cdot$ & 23.9 & 53.5 \\
\hline & B1 & $r \wedge, \wedge$ & $7, \xi$ & $\varepsilon, V$ & $11,$. & $\varepsilon q \cdot, r$ & rIo,V & 27.4 & 60.2 \\
\hline & $\mathrm{B} 2$ & $r \cdot, \Lambda$ & $v, r$ & $0, r$ & 11,7 & orr, & $r r \cdot, r$ & 30.2 & 66.3 \\
\hline & B3 & $r, \varepsilon$ & $\Lambda, \cdot$ & $7, \cdot$ & IT,. & $00 \mathrm{~V}, \mathrm{~V}$ & $r \varepsilon 1, \cdot$ & 34.5 & 70.4 \\
\hline \multirow[t]{4}{*}{ SA1 } & B0 & $r r, q$ & 0,0 & $\varepsilon, V$ & 10, & orr,r & rio,. & 26.2 & 58.7 \\
\hline & $\mathrm{B} 1$ & $r \varepsilon, \Lambda$ & $7, r$ & $0, \mathrm{~V}$ & $i v, r$ & $07 \xi, \cdot$ & $r Y V, r$ & 30.7 & 63.9 \\
\hline & B2 & $r 4,9$ & $\mathrm{v}, 1$ & $7, V$ & $r \cdot, V$ & ovv, V & rr人,. & 34.0 & 70.4 \\
\hline & B3 & $r v, \Lambda$ & $v, 7$ & $\mathrm{v}, \mathrm{r}$ & $r r, 1$ & $.09 \mathrm{~V}, \mathrm{r}$ & $r \leqslant r, \cdot$ & 39.9 & 72.4 \\
\hline \multirow[t]{4}{*}{ SA2 } & B0 & $r_{0,1}$ & $7, \cdot$ & $0, V$ & $1 \varepsilon, \Lambda$ & $001, r$ & TrY,. & 29.6 & 63.3 \\
\hline & $\mathrm{B} 1$ & $r 0,7$ & $\mathrm{~V}, \cdot$ & $7, V$ & $1 \wedge, \wedge$ & $077, \mathrm{~V}$ & $r r \Lambda, V$ & 35.5 & 68.5 \\
\hline & $\mathrm{B} 2$ & $r v, 0$ & $v, q$ & $\Lambda, \cdot$ & $r Y, V$ & ov $1, \cdot$ & $r \leqslant \wedge, \cdot$ & 42.7 & 72.9 \\
\hline & B3 & $r \wedge, \varepsilon$ & $\Lambda, 9$ & $\Lambda, r$ & $r V, T$ & $Y \cdot Y, r$ & rov,r & 49.9 & 77.9 \\
\hline \multirow[t]{4}{*}{ SA3 } & B0 & $r_{0, \Lambda}$ & 0,9 & $7, r$ & 19,7 & ov1,. & $r \vee q, r$ & 44.4 & 80.4 \\
\hline & B1 & $r 4,0$ & $\mathrm{v}, \mathrm{r}$ & $\mathrm{V}, \mathrm{V}$ & $r \varepsilon, 1$ & $0 \wedge 0, \vee$ & rrt,. & 61.3 & 96.2 \\
\hline & $\mathrm{B} 2$ & $r \Lambda, r$ & $9, r$ & $\Lambda, r$ & $r 9,7$ & $7 \cdot 0, r$ & $r \varepsilon r, v$ & 72.9 & 103.5 \\
\hline & B3 & $\varepsilon 1,1$ & $1 \cdot, 1$ & $9, \cdot$ & $r 1,0$ & $7 r r, r$ & $r 79, r$ & 81.9 & 117.8 \\
\hline \multicolumn{2}{|c|}{ LSD at 5\% } & $\cdot, 9$ & $\cdot, 7$ & $\cdot, 9$ & $\cdot, \wedge$ & $I V, r$ & $I T, V$ & 0.2 & 0.5 \\
\hline
\end{tabular}


TABLE 8 b. Effect of interaction between salicylic acid and biofertilization and sowing date on yield and its components of peanut in 2011 season .

\begin{tabular}{|c|c|c|c|c|c|c|c|c|c|}
\hline \multicolumn{2}{|c|}{ Characters } & \multirow[t]{2}{*}{\begin{tabular}{|l|}
$\begin{array}{l}\text { Plant } \\
\text { height } \\
(\mathbf{c m})\end{array}$ \\
36.0 \\
\end{tabular}} & \multirow[t]{2}{*}{$\begin{array}{l}\text { Weight } \\
\text { of } \\
\text { pods/ } \\
\text { plants } \\
\text { (g) } \\
7,1\end{array}$} & \multirow[t]{2}{*}{$\begin{array}{l}\begin{array}{l}\text { No. } \\
\text { of } \\
\text { pods/ } \\
\text { plants }\end{array} \\
0, r \\
\end{array}$} & \multirow[t]{2}{*}{\begin{tabular}{|l|}
$100-$ \\
seeds \\
weight \\
(g)
\end{tabular}} & \multirow[t]{2}{*}{$\begin{array}{l}\begin{array}{l}\text { Pod } \\
\text { yield }\end{array} \\
\text { (kg/fad) } \\
619.7\end{array}$} & \multirow[t]{2}{*}{\begin{tabular}{|l|}
$\begin{array}{l}\text { Seed } \\
\text { yield }\end{array}$ \\
(kg/fad) \\
289.7 \\
\end{tabular}} & \multirow[t]{2}{*}{\begin{tabular}{|l}
$\begin{array}{l}\text { Protein } \\
\text { yield } \\
\text { (kg/fad) }\end{array}$ \\
37.1 \\
\end{tabular}} & \multirow[t]{2}{*}{\begin{tabular}{|l|} 
Oil yield \\
(kg/fad) \\
92.9 \\
\end{tabular}} \\
\hline D1 SA0 & B0 & & & & & & & & \\
\hline & B1 & $r q, 9$ & $\Lambda, 0$ & $v, r$ & $r \varepsilon, r$ & $\mathrm{~V} \odot \Lambda, \mathrm{V}$ & roo, & \begin{tabular}{|l|}
58.3 \\
\end{tabular} & 133.5 \\
\hline & B2 & $\varepsilon r, 0$ & 9,7 & $v, r$ & $r q, \varepsilon$ & $V \vee \varepsilon$, & $r v \wedge, r$ & 69.8 & 146.4 \\
\hline & B3 & $\leq 7,1$ & 11,1 & $9,$. & $\varepsilon r, 1$ & ᄉTт,. & EOr,. & 97.7 & 183.1 \\
\hline \multirow[t]{4}{*}{ SA1 } & B0 & $r \wedge, r$ & $\mathrm{v}, \mathrm{o}$ & $0, v$ & $r r, r$ & ખ१,r & TrY,r & 41.9 & 103.1 \\
\hline & B1 & $\varepsilon 1,0$ & $9, r$ & $\Lambda, r$ & $r q, r$ & $10 \cdot, \mathrm{V}$ & rVT,r & 72.9 & 145.9 \\
\hline & B2 & $\varepsilon \varepsilon$, & 11,1 & $q, r$ & $\varepsilon r, \varepsilon$ & ᄉ०V,. & $r q \leqslant, v$ & 89.9 & 155.5 \\
\hline & B3 & $\varepsilon \vee, r$ & $1 r, r$ & $11, \mathrm{~V}$ & $\varepsilon r, q$ & $q r q, v$ & $0 . \varepsilon, r$ & 124.1 & 212.3 \\
\hline \multirow[t]{4}{*}{ SA2 } & B0 & $r q, 7$ & 9,7 & $\Lambda, \vee$ & $r v$, & V^v, & $0 \cdots, v$ & 73.6 & 180.3 \\
\hline & B1 & $\varepsilon \varepsilon, 9$ & $M r, r$ & $11, V$ & $\varepsilon \cdot, 1$ & $9 \leqslant 7, V$ & o^v, & 133.2 & 257.1 \\
\hline & B2 & $\varepsilon V, \varepsilon$ & $1 r, 9$ & $\mathrm{Ir}, \mathrm{V}$ & $\varepsilon r, 0$ & १८४ ,. & Trr,r & 167.8 & 290.1 \\
\hline & B3 & or, 9 & 19,1 & $1 \leqslant, r$ & $\varepsilon \wedge, r$ & IYYY,V & IVI,V & 194.1 & 340.6 \\
\hline \multirow[t]{4}{*}{ SA3 } & B0 & $\varepsilon 1,1$ & $\Lambda, 7$ & $\mathrm{v}, \mathrm{V}$ & rr, & VTr,. & $\sum 70$, & 67.4 & 159.5 \\
\hline & $\mathrm{B} 1$ & $\sum q, r$ & $1 \cdot, 9$ & $11, r$ & $r q, 7$ & $9 \leq \wedge, \cdot$ & $01 r, r$ & 111.9 & 210.5 \\
\hline & B2 & $\varepsilon \wedge, r$ & $1 r, 7$ & Ir,. & $\varepsilon \cdot, q$ & $907, V$ & orr, & 125.2 & 232.3 \\
\hline & B3 & $0 ., 9$ & $1 r, 9$ & $1 r, r$ & $\varepsilon \varepsilon, 7$ & $111 \leqslant,$. & ovr,. & 150.1 & 263.0 \\
\hline \multirow[t]{4}{*}{ D2 SA0 } & B0 & $r V, \varepsilon$ & 0,1 & $\varepsilon, r$ & $r V, q$ & $711, \cdot$ & 339.0 & 33.5 & 77.2 \\
\hline & B1 & ro, $\varepsilon$ & $v, \varepsilon$ & $7, \mathrm{~V}$ & $r, r$ & $u \wedge \varepsilon, \vee$ & $r \varepsilon \varepsilon,$. & 43.7 & 115.6 \\
\hline & B2 & $r v, \varepsilon$ & $\wedge, 9$ & $\mathrm{v}, \mathrm{r}$ & $r o, r$ & $V V \varepsilon, V$ & $r v v, r$ & 50.2 & 129.8 \\
\hline & B3 & $r \wedge, \wedge$ & $1 .,$. & $\Lambda, r$ & $r+\varepsilon$ & $\mathrm{V} q \varepsilon$, & $\varepsilon r \varepsilon, V$ & 65.8 & 150.3 \\
\hline \multirow[t]{4}{*}{ SA1 } & B0 & ro,r & 7,1 & $0, \mathrm{~V}$ & $r 1,9$ & $T \leqslant V, V$ & $r q \varepsilon, V$ & 36.8 & 85.8 \\
\hline & B1 & r^,. & $\Lambda, \vee$ & $\mathrm{v}, \mathrm{V}$ & $r o, r$ & VAr,r & $r r \cdot, v$ & 45.6 & 114.4 \\
\hline & B2 & $\varepsilon \cdot, r$ & $1 \cdot, 1$ & $\wedge$, & $r \Lambda, r$ & $\mathrm{~V} 90, \mathrm{~V}$ & rvo,r & 54.8 & 132.1 \\
\hline & B3 & $\varepsilon 1,9$ & $11, \varepsilon$ & $9, r$ & $\varepsilon 1$, & $\Lambda \leqslant 1, \vee$ & $\varepsilon \leqslant 0,$. & 70.3 & 163.3 \\
\hline \multirow[t]{4}{*}{ SA2 } & B0 & $r 4, \varepsilon$ & 7,7 & 7, & rr,q & $u \cdot, r$ & $r \cdot r, r$ & 41.4 & 89.8 \\
\hline & B1 & $r v, q$ & $9, V$ & $\Lambda$, & $r \varepsilon, r$ & var,. & $\varepsilon r \varepsilon,$. & 69.0 & 151.5 \\
\hline & B2 & $\varepsilon \varepsilon, r$ & $11, r$ & $9, \cdot$ & rᄉ, $\varepsilon$ & $\Lambda \| r, r$ & $\varepsilon \vee 9, v$ & 80.1 & 171.3 \\
\hline & B3 & $\varepsilon \varepsilon, 7$ & $1 r, \varepsilon$ & $1 \cdot, r$ & $\varepsilon r, 1$ & $\Delta т 1, \cdot$ & orr,. & 94.9 & 185.7 \\
\hline \multirow[t]{4}{*}{ SA3 } & B0 & $r \wedge, 0$ & $v, r$ & $\mathrm{v}, \mathrm{v}$ & $r v, \Lambda$ & $V v \cdot, \cdot$ & $\varepsilon q 4, \cdot$ & 72.9 & 174.5 \\
\hline & B1 & $\varepsilon \cdot, r$ & $1 \cdot, 0$ & $9, r$ & $r_{\Lambda, \lambda}$ & $q \nmid \Lambda, r$ & $\Delta \wedge V, r$ & 118.6 & 239.6 \\
\hline & B2 & $\varepsilon 0, \varepsilon$ & $11, \wedge$ & $11, v$ & $\varepsilon r, 0$ & $90 \cdot, r$ & $T 1 \mathrm{~V}, \mathrm{~V}$ & 147.0 & 268.7 \\
\hline & B3 & $\leqslant \vee, \uparrow$ & $1 r, 0$ & $1 \leqslant,$. & $\sum \uparrow, \wedge$ & IYKA,. & $707, r$ & 169.3 & 299.3 \\
\hline \multirow[t]{4}{*}{ D3 SA0 } & B0 & $r \varepsilon, \varepsilon$ & 0,1 & $r, v$ & $1 \cdot, \varepsilon$ & $0 . Y, V$ & $r \mid \leqslant, r$ & 25.5 & 56.1 \\
\hline & B1 & $r \cdot, 1$ & $7, V$ & $0, v$ & $11, r$ & oor,r & $r r q, V$ & 29.4 & 64.7 \\
\hline & B2 & $r, v$ & $v, 0$ & 7, & $11, v$ & ovo,. & $r \leqslant 1, V$ & 31.9 & 71.1 \\
\hline & B3 & $r r, o$ & $\Lambda, 1$ & $7, \mathrm{~V}$ & $1 Y, \varepsilon$ & $0 \wedge \cdot, \cdot$ & $r \leqslant V, V$ & 35.4 & 73.8 \\
\hline \multirow[t]{4}{*}{ SA1 } & B0 & $r \varepsilon, r$ & $0, V$ & $0,$. & 10,1 & 001, & YYY,r & 27.1 & 60.7 \\
\hline & B1 & $r_{0, \varepsilon}$ & $\mathrm{T}, \mathrm{V}$ & $0, v$ & $|v|$, & $O V \varepsilon$, & rrr,r & 31.6 & 65.7 \\
\hline & B2 & $r \wedge, r$ & $\mathrm{v}, \Lambda$ & $\mathrm{v}$, & $r I, r$ & ONV,V & $r \varepsilon .,$. & 34.3 & 71.2 \\
\hline & B3 & $r q, 1$ & $\Lambda, \wedge$ & $\Lambda, r$ & $r r, r$ & T.r, & $r \leqslant \wedge, \cdot$ & 41.4 & 74.9 \\
\hline \multirow[t]{4}{*}{ SA2 } & B0 & $r_{4}$, & 7,9 & $0, v$ & 10, & otv, & rro,r & 30.4 & 63.9 \\
\hline & B1 & $r 4, r$ & $v, \varepsilon$ & $\mathrm{v}$, & 19,7 & ONY,V & $r \leqslant 1,$. & 37.1 & 69.4 \\
\hline & B2 & rᄉ, & $\Lambda, 0$ & $\wedge$, & $r r, r$ & oqr,V & ros,r & 43.9 & 77.1 \\
\hline & B3 & $\varepsilon \cdot, v$ & 9,7 & $\Lambda, r$ & $r \Lambda, r$ & $711, r$ & ruv,r & 51.8 & 82.3 \\
\hline SA3 & B0 & $r o, r$ & $T, Y$ & 7, & $r \cdot, r$ & ovi,r & r८९,. & 42.8 & 83.8 \\
\hline
\end{tabular}

Egypt. J. Agron. 35, No. 1 (2013) 


\begin{tabular}{|c|c|c|c|c|c|c|c|c|c|}
\hline & B1 & $r 4, \mathrm{~V}$ & $v, 7$ & $v, r$ & $r \varepsilon, 7$ & $09 r, r$ & rro,v & 64.5 & 100.0 \\
\hline & B2 & $r q, 7$ & 9,9 & $\Lambda, r$ & $r q, V$ & $T \cdot V,$. & $r \varepsilon 7, v$ & 74.2 & 106.1 \\
\hline & B3 & $\varepsilon 1,9$ & $1 \cdot, \varepsilon$ & $9, \cdot$ & 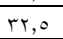 & $T \varepsilon \cdot, V$ & $r v \cdot, v$ & 84.5 & 120.1 \\
\hline LSD at $5 \%$ & & 1.4 & $\cdot, \mathrm{V}$ & $\cdot, 9$ & $1, v$ & $1 \Lambda, \varepsilon$ & $1 r, v$ & 0.4 & 0.7 \\
\hline
\end{tabular}

Effect of treatments on the microbial determinations

Data in Table 9 showed that the total microbial counts at rhizosphere of inoculated plants were significantly higher in mixed treatments than in single inoculants. The highest significant increases was recorded with sowing date $20^{\text {th }}$ April, salicylic acid concentration 200ppm and mixed inoculation treatment, (Rhizobia as base treatment, PDB+ Psudomonase, being $217 \times 10^{5} \mathrm{cfu} / \mathrm{g}$ dry soil) compared with control. These results in compatible with the finding of El-Wakeil \& El-Sebai (2007) who reported that total microbial count was significantly higher in mixed inoculants' strains than in single inoculants at rhizosphere of inoculated faba bean plants.

TABLE 9. Main effect of salicylic acid, biofertilization and sowing date, on some microbial characters in the two growing seasons.

\begin{tabular}{|c|c|c|c|c|c|c|c|c|c|c|}
\hline \multirow{3}{*}{ Tharacters } & \multicolumn{6}{|c|}{ Microbial determinations } & \multicolumn{4}{|c|}{ Nodule characteristics } \\
\hline & \multicolumn{2}{|c|}{$\begin{array}{l}\text { Total microbial } \\
\text { in soil }\end{array}$} & \multicolumn{2}{|c|}{$\begin{array}{c}\text { PDB count in } \\
\text { soil }\end{array}$} & \multicolumn{2}{|c|}{$\begin{array}{c}\text { Pseudomonas } \\
\text { count in soil }\end{array}$} & \multicolumn{2}{|c|}{$\begin{array}{c}\text { No. of nodule / } \\
\text { plant }\end{array}$} & \multicolumn{2}{|c|}{$\begin{array}{c}\text { Nodule dry } \\
\text { weight } \\
\text { mg/plant }\end{array}$} \\
\hline & 2010 & 2011 & 2010 & 2011 & 2010 & 2011 & 2010 & 2011 & 2010 & 2011 \\
\hline \multicolumn{11}{|c|}{ Sowing date } \\
\hline $20^{\text {th }}$ April & 161.9 & 177.2 & 57.9 & 70.6 & 25.9 & 34.3 & $20.2 \mathrm{a}$ & 21.4 & 71.8 & 73.7 \\
\hline $15^{\text {th }}$ May & 143.9 & 161.3 & 53.5 & 66.7 & 25.8 & 33.5 & $18.8 \mathrm{a}$ & 19.2 & 51.5 & 54.9 \\
\hline $10^{\text {th }}$ June & 133.6 & 148.7 & 42.3 & 49.2 & 25.8 & 31.6 & $13.6 b$ & 14.3 & 36.4 & 39.9 \\
\hline LSD5\% & 5.1 & 2.1 & 1.7 & 2.6 & 0.9 & 1.4 & 3.6 & 1.6 & 5.4 & 5.2 \\
\hline \multicolumn{11}{|c|}{ Salicylic acid } \\
\hline Control & 69.4 & 78.1 & 26.4 & 32.1 & 14.1 & 18.5 & 11.2 & 12.1 & 37.5 & 40.1 \\
\hline $100 \mathrm{ppm}$ & 148.3 & 162.7 & 59.3 & 74.3 & 20.6 & 25.5 & 17.9 & 17.3 & 45.9 & 49.4 \\
\hline $150 \mathrm{ppm}$ & 170.0 & 191.9 & 39.4 & 47.9 & 32.8 & 40.6 & 18.8 & 19.7 & 56.8 & 59.6 \\
\hline $200 \mathrm{ppm}$ & 198.3 & 216.9 & 79.8 & 94.3 & 36.1 & 47.9 & 22.0 & 24.0 & 72.6 & 75.6 \\
\hline LSD5\% & 3.1 & 2.1 & 1.3 & 1.6 & 1.1 & 1.3 & 4.0 & 0.7 & 3.2 & 3.4 \\
\hline \multicolumn{11}{|c|}{ Biofertilization } \\
\hline Control Br & 122.9 & 139.4 & 39.5 & 50.1 & 21.4 & 30.8 & 14.2 & 13.8 & 38.1 & 40.5 \\
\hline PDB & 146.7 & 161.5 & 47.7 & 58.6 & 25.1 & 32.5 & 15.3 & 15.8 & 49.4 & 51.7 \\
\hline PS & 154.6 & 170.9 & 57.4 & 69.5 & 27.9 & 33.1 & 19.6 & 21.6 & 55.8 & 59.9 \\
\hline $\begin{array}{l}\operatorname{mix}(\mathrm{PDB}+ \\
\mathrm{BS})\end{array}$ & 161.8 & 177.8 & 60.4 & 70.4 & 28.9 & 36.2 & 20.8 & 22.3 & 69.4 & 72.6 \\
\hline LSD5\% & 2.9 & 1.5 & 1.9 & 1.9 & 1.2 & 1.0 & 4.0 & 0.9 & 1.9 & 1.7 \\
\hline
\end{tabular}

It was clear from the data represented in Table 8 that inoculation with rhizobia and PDB+ Pseudomonas stimulated the activity and growth of phosphate dissolving bacteria in rhizosphere area of inoculated plant. The highest density of PDB being $96.4 \times 10^{2}$ cfu /g dry soil recorded with mixed treatment Bradyrhizobia as base treatment, PDB+ Psudomonase. These results are in agreements with Gyanshwar et al. (2002) who confirmed that increase in PDB count leads to corresponding increase in the availability and mobility of phosphorous and other plant nutrients from soil to plant through production of organic acids. These effects were reflected on increasing of plant growth, crop production and oil yield 
Also, mixed inoculation with rhizobia and PDB+ Pseudomonas stimulated the activity and growth of Pseudomonas in rhizosphere area of inoculated plants. The highest density of Pseudomonas being $49.3 \times 10^{2} \mathrm{cfu} / \mathrm{g}$ dry soil recorded with mixed treatment. These results are in agreement with Usha (2003) and El-shazly (2010) who reported that Pseudomonas strain may be helpful as plant growth promoting bacteria and bio-control agent. Also, Pseudomonas has tremendous importance due to its widespread distribution in soil. This may be due to utilizing a wide range of organic substances as carbon or nitrogen sources.

Application of biofertilizers is an acceptable approach for higher yield with good quality and safe for human consumption. Our results show that either single or mixed inoculates gave positive response to the studied parameters. This response was accompanied by significant increase in soil microbial determinations and nodule characteristics (Table 9) thus mixed inoculation treatment is the best compared with single inoculation treatment. These findings is on the same line with those obtained by Monibe et al. (1998) who found that single inoculation of rhizoidal performed lower in terms of $\mathrm{N}_{2}$ fixation and $\mathrm{N}$ accumulation capacities as compared with the mixture of this strain with some other strains. Also, Moawad et al. (2004) mentioned that the rhizobia inoculation showed a positive response to inoculation in terms of nodule numbers and dry weight and also enhanced growth of Phaseolus bean and $\mathrm{N}$ values compared to control. The mixed inoculation treatment of rhizobia and PDB + Pseudomonas enhanced all tested parameters more than single inoculation treatment with PDB or Pseudomonas compared with control. This inspection was confirmed by Moawad \& Abd El-Rahim (2002) who reported that biofertilizers treatments applied increased growth parameters and NPK contents

In respect to effect of interaction of sowing date and salicylic acid on microbial determinations (Fig. 2a, 2b and 2c) represented data showed that sowing date at April $20^{\text {th }}$ recorded highest microbial counts (Total, PDB and Pseudomonas counts) followed by $2^{\text {nd }}$ sowing date. Also, salicylic acid concentrations exhibited pronounced effect on microbial counts, SA3 (200ppm) recorded highest microbial counts compared to other concentrations.

Regarding to effect of interaction of sowing date and biofertilizations on microbial determinations (Fig. 3a, 3b and 3c) represented data showed that sowing date at April $20^{\text {th }}$ recorded highest microbial counts (Total microbial, PDB and Pseudomonas counts) followed by $2^{\text {nd }}$ sowing date. Also, mixed biofertilization treatments had a stimulatory effect on microbial counts, which recorded highest microbial counts compared to other biofertilization treatments.

In order to study the effect of interaction between salicylic acid concentrations and biofertilization on microbial counts in peanut rhizosphere, obtained data representd in Fig. 4a, 4b and 4c revealed that SA3 (200ppm) recorded highest microbial counts compared to other concentrations. Also, mixed biofertilization treatments had a stimulatory effect on microbial counts, which recorded highest microbial counts compared to other biofertilization treatments.

Egypt. J. Agron. 35, No. 1 (2013) 
The effect of interaction between salicylic acid foliar application, biofertilization and sowing date was significant for microbial determination and nodule characteristics (Table 10). The maximum value of Total microbial counts, PDB counts , Psedomonas counts, Number of Nodule /plant and nodule dry weight $\mathrm{mg} /$ plant were obtained at growing seasons 2010 and 2011, from plants sown on April $20^{\text {th }}$ and supplied with 200 ppm salicylic acid and mixed biofertilization treatments

It could be concluded that sowing of peanut cv., Giza, 6 may be completed from April $20^{\text {th }}$ to May $15^{\text {th }}$ with foliar spraying by salicylic acid at rate $150-200$ ppm and mix two types of biofertilization (PDB + Pseudomonas) in order to raise a healthy and good peanut crop and ultimately get highest yield.

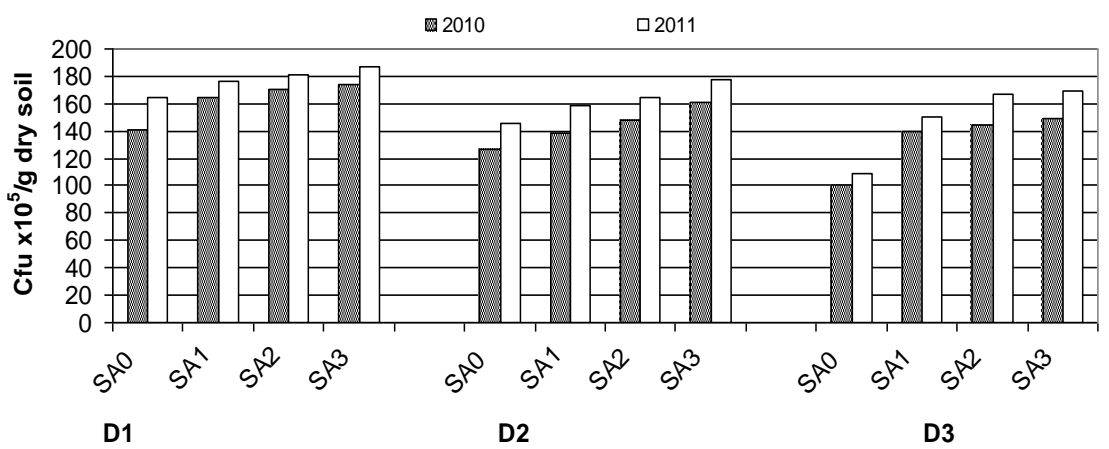

Fig. 2a. Interaction sowing date and salicylic acid on total microbial counts at two growing seasons.

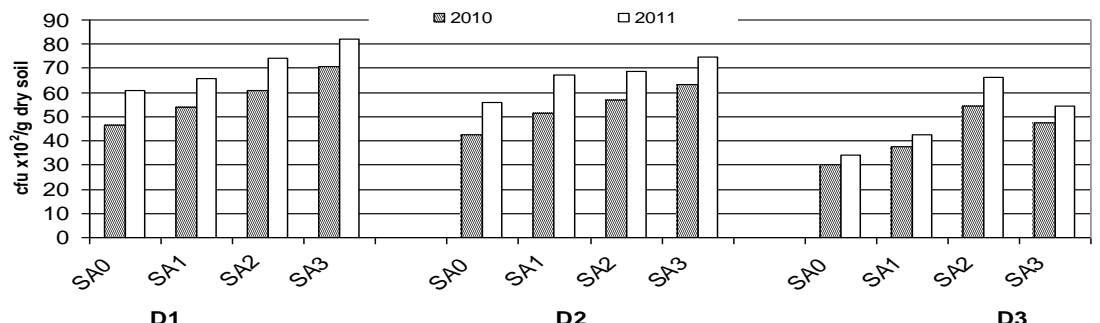

Fig. 2b. Interaction sowing date and salicylic acid on PDB at two growing seasons .

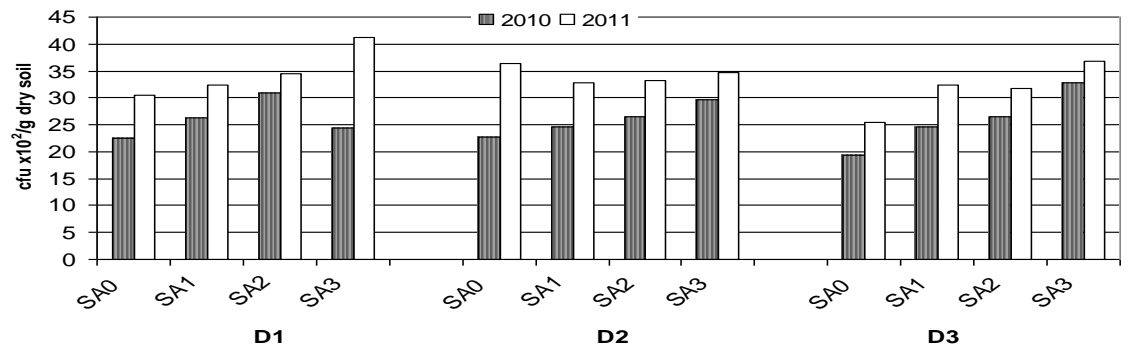

Fig. 2c. Interaction sowing date and salicylic acid on Pseudomonas counts at two growing seasons . 


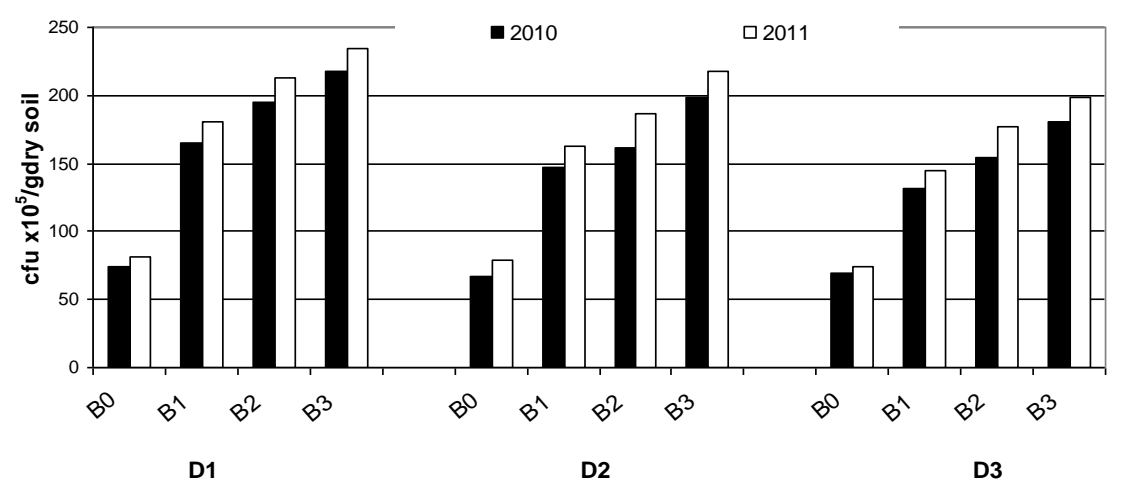

Fig. 3 a. Interaction sowing date and biofertilization on total microbial counts at two growing seasons .

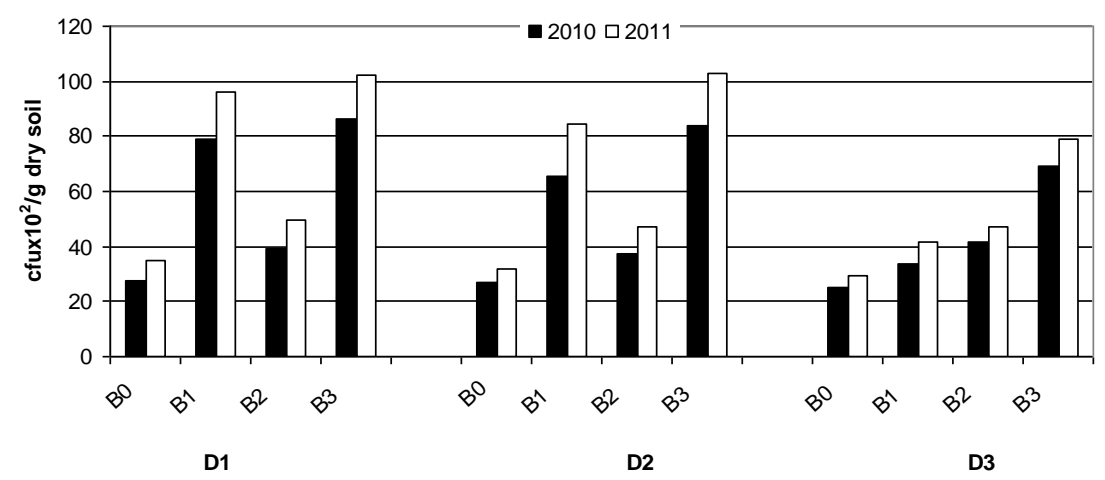

Fig. 3 b. Interaction sowing date and biofertilization on PDB at two growing seasons .

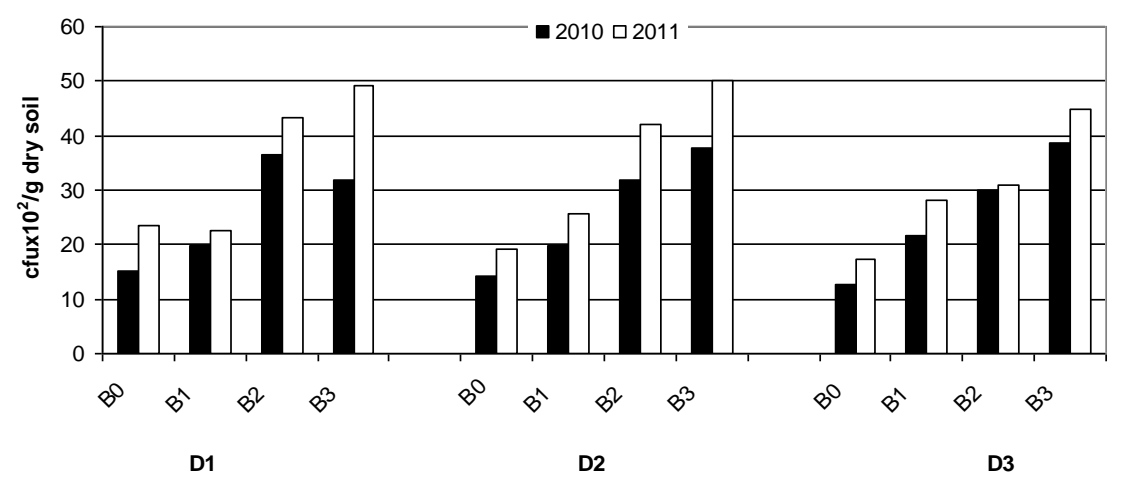

Fig. 3c. Interaction sowing date and biofertilization on Pseudomonas counts at two growing seasons .

Egypt. J. Agron. 35, No. 1 (2013) 


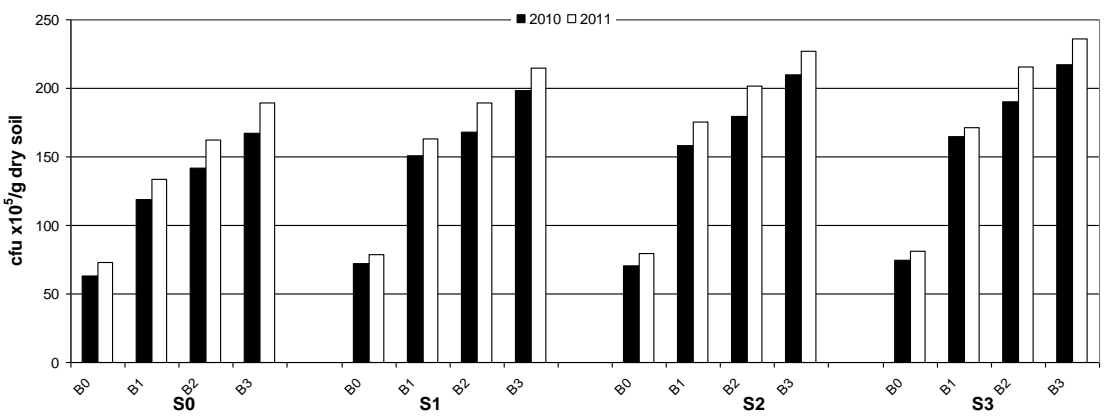

Fig.4a. Effect of interaction between salicylic acid and biofetilization on total microbial counts at two growing seasons .

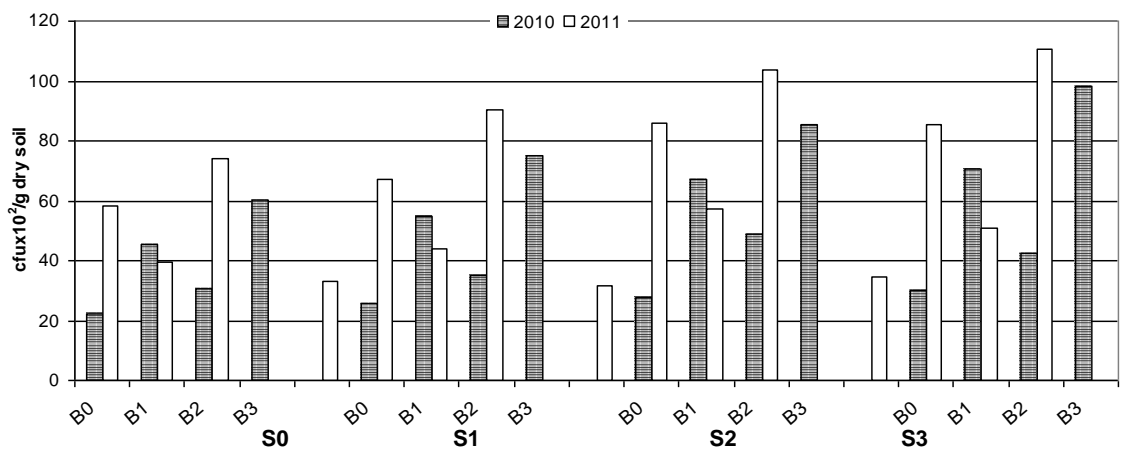

Fig. 4b. Effect of interaction between salicylic acid and biofetilization on PDB at two growing seasons .

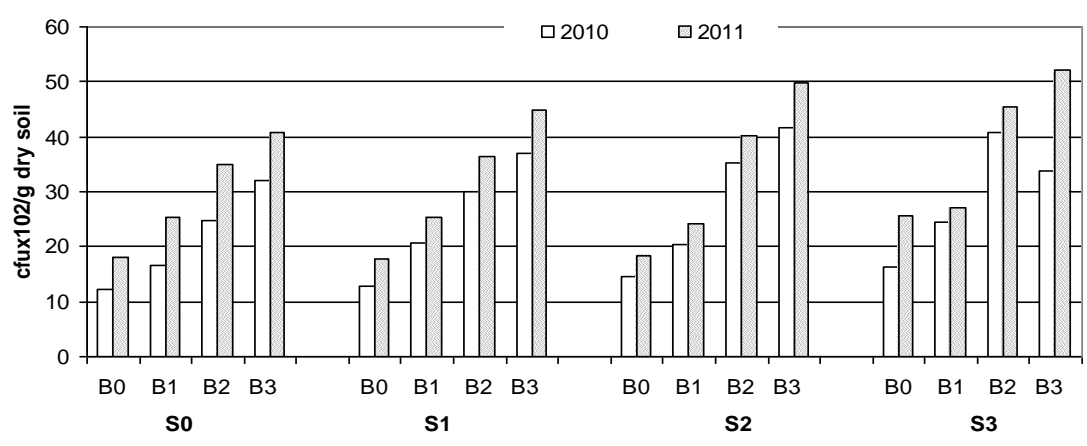

Fig. 4c. Effect of interaction between salicylic acid and biofetilization on Pseudomonas counts at two growing seasons .

$\mathrm{TC}=$ Total microbial in soil, $\mathrm{PDB}=\mathrm{PDB}$ count in soil and $\mathrm{Ps}=$ Pseudomonas count in soil.

$\mathrm{SA} 0=$ Zero salicylic acid, SA1, SA2 and SA3 $=100,150$ and $200 \mathrm{ppm}$ salicylic acid $\mathrm{B} 0=\mathrm{Br}$ $\mathrm{B} 1=\mathrm{PDB}$ bacteria, $\mathrm{B} 2=\mathrm{PS}$ bacteria and $\mathrm{B} 3=\operatorname{mix}(\mathrm{PDB}+\mathrm{BS})$ and $\mathrm{D} 1=20^{\text {th }}$ April, D2 $=15^{\text {th }}$ May and $\mathrm{D} 3=10^{\text {th }} \mathrm{Jun}$ 
TABLE 10.Effect of interaction between salicylic acid and biofertilization and sowing date on microbial determinations and nodule characteristics of peanut at two growing seasons

\begin{tabular}{|c|c|c|c|c|c|c|c|c|c|c|c|c|}
\hline \multirow{3}{*}{\multicolumn{3}{|c|}{ Treatments }} & \multicolumn{6}{|c|}{ Microbial determinations } & \multicolumn{4}{|c|}{ Nodule characteristics } \\
\hline & & & \multicolumn{2}{|c|}{ T.microbial } & \multicolumn{2}{|c|}{ PDB } & \multicolumn{2}{|c|}{ Pseudomonas } & \multicolumn{2}{|c|}{$\begin{array}{c}\text { No.of nodule / } \\
\text { plant }\end{array}$} & \multicolumn{2}{|c|}{$\begin{array}{c}\text { Nodule dry } \\
\text { weight } \\
\text { mg/plant }\end{array}$} \\
\hline & & & 2010 & 2011 & 2010 & 2011 & 2010 & 2011 & 2010 & 2011 & 2010 & 2011 \\
\hline \multirow{16}{*}{$\bar{a}$} & \multirow{4}{*}{ 密 } & $\overline{\mathrm{B} 0}$ & 66.3 & 77.0 & 24.3 & 32.0 & 13.0 & 17.7 & 9.7 & 11.0 & 29.6 & 30.7 \\
\hline & & B1 & 142.0 & 158.0 & 58.0 & 79.0 & 16.0 & 20.7 & 13.0 & 14.0 & 36.6 & 37.8 \\
\hline & & B2 & 164.3 & 193.3 & 32.3 & 44.7 & 27.7 & 39.7 & 13.3 & 15.7 & 48.2 & 49.7 \\
\hline & & B3 & 193.0 & 227.7 & 71.0 & 87.3 & 32.7 & 44.0 & 16.0 & 18.0 & 62.6 & 63.9 \\
\hline & \multirow{4}{*}{ 茫 } & B0 & 79.3 & 81.7 & 26.3 & 35.0 & 14.0 & 17.7 & 9.7 & 11.4 & 35.1 & 36.5 \\
\hline & & B1 & 168.7 & 184.0 & 72.0 & 88.7 & 19.7 & 22.0 & 14.0 & 16.0 & 49.5 & 51.8 \\
\hline & & B2 & 194.7 & 214.0 & 38.7 & 46.7 & 33.0 & 41.7 & 15.7 & 16.0 & 52.2 & 54.0 \\
\hline & & B3 & 213.3 & 223.3 & 79.0 & 93.0 & 38.0 & 47.7 & 20.3 & 21.7 & 77.3 & 78.2 \\
\hline & \multirow{4}{*}{ 访 } & B0 & 72.7 & 81.3 & 28.7 & 35.3 & 16.7 & 19.3 & 18.7 & 18.7 & 44.5 & 49.2 \\
\hline & & B1 & 179.3 & 188.0 & 87.3 & 103.3 & 21.7 & 24.3 & 26.3 & 26.7 & 73.9 & 76.3 \\
\hline & & B2 & 207.7 & 219.0 & 39.0 & 50.3 & 39.7 & 43.3 & 27.3 & 30.3 & 85.5 & 87.2 \\
\hline & & B3 & 223.0 & 237.7 & 87.7 & 108.0 & 45.7 & 50.7 & 37.7 & 38.7 & 113.4 & 115.4 \\
\hline & \multirow{4}{*}{$\sum_{\infty}^{2}$} & B0 & 87.0 & 83.3 & 30.3 & 38.3 & 18.3 & 39.0 & 16.3 & 16.0 & 66.0 & 67.9 \\
\hline & & $\mathrm{B} 1$ & 171.7 & 194.0 & 98.7 & 114.7 & 22.3 & 23.3 & 21.0 & 24.3 & 112.3 & 114.1 \\
\hline & & B2 & 217.0 & 223.7 & 46.7 & 55.3 & 45.3 & 48.3 & 26.3 & 27.0 & 119.2 & 120.9 \\
\hline & & $\bar{B} 3$ & 234.7 & 248.7 & 107.3 & 120.0 & 11.7 & 54.7 & 35.3 & 37.0 & 142.3 & 146.0 \\
\hline \multirow{16}{*}{$\tilde{\Omega}$} & \multirow{4}{*}{ 离 } & B0 & 61.3 & 74.7 & 20.7 & 28.3 & 14.3 & 23.0 & 9.3 & 10.3 & 29.1 & 30.2 \\
\hline & & B1 & 131.3 & 145.3 & 50.7 & 67.3 & 21.3 & 34.3 & 12.3 & 13.7 & 38.1 & 39.6 \\
\hline & & B2 & 143.0 & 168.0 & 29.3 & 41.3 & 24.3 & 42.3 & 16.3 & 16.0 & 42.7 & 51.7 \\
\hline & & B3 & 171.7 & 193.3 & 69.3 & 86.3 & 30.7 & 45.7 & 18.3 & 18.0 & 47.3 & 55.7 \\
\hline & \multirow{4}{*}{ 芯 } & B0 & 67.0 & 79.7 & 27.3 & 35.7 & 13.3 & 17.7 & 10.0 & 11.3 & 37.8 & 40.1 \\
\hline & & B1 & 144.3 & 161.0 & 61.7 & 81.0 & 17.7 & 22.3 & 15.0 & 17.3 & 43.6 & 45.6 \\
\hline & & B2 & 152.0 & 177.7 & 36.7 & 49.3 & 31.0 & 41.0 & 15.7 & 20.0 & 54.3 & 57.5 \\
\hline & & B3 & 191.3 & 213.7 & 79.7 & 103.0 & 36.3 & 50.7 & 19.7 & 24.3 & 62.2 & 64.0 \\
\hline & \multirow{4}{*}{$\underset{\pi}{2}$} & B0 & 69.0 & 80.0 & 28.3 & 29.7 & 13.3 & 17.3 & 10.3 & 12.3 & 39.3 & 42.0 \\
\hline & & B1 & 152.3 & 170.7 & 68.7 & 88.0 & 19.7 & 23.3 & 17.0 & 21.3 & 42.8 & 43.0 \\
\hline & & B2 & 163.3 & 184.3 & 40.7 & 47.0 & 33.7 & 41.3 & 20.0 & 23.7 & 56.3 & 59.3 \\
\hline & & B3 & 209.3 & 224.3 & 90.3 & 110.0 & 39.0 & 50.7 & 19.7 & 27.0 & 71.3 & 73.2 \\
\hline & & B0 & 72.7 & 81.0 & 30.3 & 33.7 & 15.3 & 18.7 & 11.7 & 13.3 & 48.3 & 50.2 \\
\hline & 2 & B1 & 162.3 & 173.7 & 82.0 & 101.7 & 21.0 & 23.3 & 21.7 & 24.0 & 63.0 & 64.6 \\
\hline & 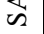 & B2 & 188.0 & 215.0 & 43.0 & 51.3 & 38.3 & 44.3 & 21.3 & 24.3 & 68.0 & 72.9 \\
\hline & & B3 & 223.0 & 238.3 & 97.0 & 113.0 & 44.3 & 53.0 & 28.3 & 30.3 & 78.6 & 85.9 \\
\hline & & B0 & 63.3 & 67.7 & 21.7 & 26.7 & 9.0 & 13.3 & 8.0 & 8.3 & 21.5 & 22.4 \\
\hline & 우 & B1 & 83.7 & 97.0 & 28.0 & 29.0 & 12.7 & 21.3 & 9.7 & 9.3 & 29.7 & 31.3 \\
\hline & 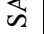 & B2 & 118.7 & 124.3 & 30.3 & 33.0 & 22.7 & 29.7 & 11.0 & 8.7 & 30.5 & 32.7 \\
\hline & & B3 & 137.0 & 146.3 & 40.0 & 48.0 & 32.7 & 37.7 & 13.3 & 15.3 & 33.6 & 35.1 \\
\hline & & B0 & 70.0 & 74.7 & 23.0 & 29.0 & 11.3 & 18.3 & 9.7 & 9.3 & 29.6 & 34.0 \\
\hline & 7 & B1 & 140.7 & 144.0 & 30.0 & 31.3 & 24.3 & 31.3 & 11.0 & 11.3 & 31.8 & 37.6 \\
\hline & 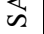 & B2 & 157.3 & 176.3 & 30.3 & 35.3 & 26.3 & 35.7 & 13.7 & 14.3 & 35.1 & 46.8 \\
\hline$m$ & & B3 & 190.3 & 207.7 & 67.3 & 75.3 & 36.7 & 44.3 & 16.3 & 16.7 & 42.2 & 44.1 \\
\hline Q & & B0 & 69.3 & 78.0 & 26.7 & 29.7 & 14.3 & 18.3 & 10.3 & 7.7 & 37.8 & 42.0 \\
\hline & V & B1 & 143.3 & 166.7 & 45.7 & 67.0 & 20.0 & 25.0 & 13.3 & 13.7 & 32.0 & 34.8 \\
\hline & 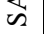 & $\mathrm{B} 2$ & 167.7 & 200.7 & 67.3 & 74.7 & 32.0 & 36.3 & 16.3 & 17.0 & 36.3 & 40.3 \\
\hline & & B3 & 198.3 & 219.7 & 78.0 & 93.0 & 40.0 & 47.7 & 18.7 & 19.3 & 48.3 & 51.9 \\
\hline & & B0 & 73.0 & 78.0 & 29.3 & 31.7 & 15.7 & 19.3 & 11.0 & 9.3 & 38.8 & 40.5 \\
\hline & & B1 & 159.3 & 169.7 & 31.3 & 40.0 & 30.3 & 34.7 & 15.7 & 16.3 & 39.4 & 40.1 \\
\hline & 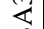 & $\mathrm{B} 2$ & 171.3 & 207.0 & 38.3 & 46.3 & 39.3 & 44.0 & 18.7 & 20.0 & 42.3 & 47.3 \\
\hline & & B3 & 195.0 & 221.7 & 91.0 & 99.0 & 45.7 & 49.0 & 20.3 & 21.7 & 53.0 & 57.0 \\
\hline L. & Dat & $5 \%$ & 11.271 & 7.5071 & 4.795 & 5.8584 & 4.112 & 4.6718 & n.s & 2.5171 & 7.0076 & 6.1204 \\
\hline
\end{tabular}

Egypt. J. Agron. 35, No. 1 (2013) 


\section{References}

Ahmed, N., Rahim, M. and Khan, U. (2007) Evaluation of varieties sowing dates for groundnut under Agro-Ecological conditions of Malakand division. Gomel. University J. Res. 23(2)1-4.

Ali, E.A. and Mahmoud, A.M. (2012) Effect of foliar spray by different salicylic acid and zinc concentrations on seed yield and yield components of mungbean in sandy soils. Asian. J. Crop Sci. ISSN 1994-7879.

Amutha, R., Muthulaksmi, S., Balyrani, W., Indira, K. and Mareeswari, P. (2007) Alleviation of high temperature stress in sunflower (Helianthus annus L.) by plant growth regulators and chemicals. Res. J. Agric. Biological Sci. 3(12),1658-1662.

A.O. A. C. (1980) "Association of Official Methods of Analysis". 13 $3^{\text {th }}$ ed., Washington, D.C.

Bai, Y., Souleimanov, A. and Smith, D.I. (2002) An inducible activator produced by Serratia proteamaclans strain and its soybean growth promoting activity under greenhouse conditions. J. Exp. Bot. 53 , 149-502.

Badawi, F.S., Biomy, A.M.M. and Desouky, A.H. (2011) Peanut plant growth and yield as influenced by co-inoculation with Bradyrhizobium and some rhizo-microorganisms under sandy loam soil conditions. J. Agric. Sci.56,17-25.

Bala, H.M., Ogunlela, V.B., Kuchinda, N.C. and Tanimu, B. (2011) Response of two groundnuts (Arachis hypogaea L.) varieties to sowing date and NPK fertilizer rate in a Semi-Arid environment: Yield and yield attributes. Asian J. Crop Sci. 3(3) 130-140.

Baldwin, J. (2005) Seeding rate, row patterns and planting dates. Peanut Home Page Available at http://WWW.caes. uga.edu/ commodities/ field crops/ peanut/ index. html. (Verifield on 26 August 2008).

Barrow, G.L and Velthan, R.K.A. (1993) "Cown \& Steel's, Mannual for the Identification of Medical Bacteria". Cambridge Univ. Press.

Baset Mia, M.H and Shamsuddin, Z. H. (2010) Rhizobium as a crop enhancer and biofertilizer for increased cereal production. African Journal of Biotechnology, 9(37), 6001-6009.

Bell, M. (2001) Effect of sowing date on growth and development of irrigated peanuts, early bunch, in a monsoonal tropical environment. Australian J. Agric. Res. 37(4), 361-373.

Bergey's Manual of Systemic Bacteriology (1984) Vol. (1), Krieg,N.R. and Holt, J.G. (Editor). Genus Azospirillum. p. 94-103 and Genus Azotobacter p. 225-229. (II) p. 1104-1139. Sneath, Ph.D. (Editor). Genus Bacillus subtilis. Williams, Wilkins, Baltimore, USA.

Chapman, H.D. and Pratt, R.F. (1978) "Methods Analysis for Soil, Plant and Water". Univ. California. Div. Agric. Sci. pp.16-38. 
Cherry, J. H. (1973) "Molecular Biology of Plants Test-Manual". Colombia Univ. Press, New York and London pp.68-71.

Choudhury, A.T.M.A. and Kennedy. I.R. (2004) Prospects and potentials for system of biological nitrogen fixation in sustainable rice production. Biol. Fertil. Soils. 39, 219-227.

Cochran, W.G. (1950) "Estimation of Bacterial Densities by Means of the Most Probable Number". Biometrics, 6, 105-115.

Dat, J. F., Lopez-Delgado, H., Foyer, C.H. and Scott, I.M. (2000) Effect of salicylic acid on oxidative stress and thermo tolerance in tobacco. J. Plant Physiol. 156, 659-65.

El-Saidy, Aml E.A. and Abd El-Hai, K.M. (2011) Alleviation of peanut seed deterioration during storage using biotic and abiotic agents. Res. J. Seed Sci. ISSN 1819-3552/Dol: 10.3923/rjss, p: 1-13.

El-shazly, Mona M. (2010) Employment of some effective microorganisms in improving sandy soil properties and productivity. PhD. Thesis, Fac. Sci., Al-Azhar Univ., Cairo, Egypt.

El-Shraiy, A.M. and Hegazi, A.M. (2009) Effect of acetylsalicylic acid, indole-3-bytric acid and giberelic acid on plant growth and yield of pea (Pisum sativum L.). Aust. J. Basic Applied Sci. 3, 3514-3523.

El-Wakeil, N.E. and El-Sebai, T.N. (2007) Role of biofertilizer on faba bean growth yield, and its effect on bean aphid and the associated predators. Res. J. Agric. Biol. Sci. 3 (6) 800-807.

Frimpong, A. (2004) Characterzation of groundnut (Arachis hypogaea L.) in Northern Ghana. Pakistan. J. Biol. Sci. 7, 838-842.

Gomez, K.A. and Gomez, A.A. (1984) "Statistical Procedures for Agricultural Research". $2^{\text {nd }}$ ed., John Wiley and Sons Inc., New York: pp.95-109.

Gyaneshwar, P., Kumar, G.N., Parekh, L. J. and Poole, P.S. (2002) Role of soil microorganisms in improving P nutrition of plants. Plant and Soil, 245,83-93.

Hatam, M. and Abbasi, G. Q. (1994) Oil Seed Crops. In: "Crop Production", Elena Bashir and Robyn Bantel (Ed.), Pub. National Book Foundation, pp. 352-353.

Hussein, M.M., Balbaa, L.K. and Gaballah, M.S. (2007) Salicylic acid and salinity effects on growth of maize plants. Res. J. Agric. Biol. Sci. 3(4),321-328.

Janda, T., Szalai, Horvth, G. and Plaldi, E. (2007) "Role of Salicylic Acid in the Induction of Abiotic Stress Tolerance". Springer Netherlands: pp.91-150.

Jarlier, V., Fosse, T. and Philipon, A. (1996) Antibiotic susceptibility in aerobic Gramnegative bacilli isolated in intensive care units in 39 French teaching hospitals (ICU study). Intensive Care Mod. 22, 1057-1065. 
Jeyabal, A. and Kupuswamy, G. (2001) Recycling of organic wastes for the production of vermicompost and its response in rice-legume cropping system and soil fertility. Eur. J. Agron. 15,153-170.

Kaci, Y., Heyraud, A., Mohamed, B. and Heulin, T. (2005) Isolation and identification of an EPS-producing Rhizobium strain from arid soil (Algeria): Characterization of its EPS and the effect of inoculation on wheat rhizosphere soil structure. Research in Microbiology, 156, 522-531.

Kasai, S. F., Paulo, M. E., Godoy, D. J. I. and Nagai, V. (1999) Influence of sowing time on growth, productivity and other yield characters of peanut cultivars in the Alta Paulista Region. State of Sao Paulo, Aragonite v.58 n.1 Campinas pp.95-107.

Kennedy, I.R., Choudhury, A.T. and Kecskés, M.L. (2004) Non-symbiotic bacterial diazotrophs in crop-farming systems: can their potential for plant growth promotion be better exploited. Soil Biol. Biochem. 36,1229-1244.

Khan, W., Pelrithiven, R. and Smith, A. (2003) Photosynthetic responses of corn and soybean to foliar application of salicylates. J. Plant Physiology.160, 485-492.

King, E. O., Ward, M.K. and Raney, O. E. (1954) Two simple media for the demonstration of pyocyanin and fluorescein. J. Lab. Clin. Med. 44, 301-307.

Laurence, R.C.N. (1983) Effects of sowing date, spatial arrangement and population on yield and kernel weight of irrigated Virginia Bunch peanuts. Austr. J. Exper. Agric., Animal Husbandry 23(121), 178-180.

Marek-Kozaczuk, M. and Skorupska, A. (1997) Physiological parameters influencing the production of siderophore by PGPR Pseudomonas sp. strain 267: ActaMicrobiologica-Polonica, 46 (2), 157-165.

Mehana, T.A. and Farag, F.M. (2000) Influence of phosphate dissolving microorganisms and elemental sulphur on phosphorous and micronutrient availability in a calcareous soil treated with rock phosphate. J. Agric. Sci. Mansoura Univ. 25, 2983-2993.

Metwally, A., Finkemeier, I. Georgi, M. and Dietz, K. (2003) Salicylic acid alleviates the cadmium toxicity in barley seedlings. Plant Physiol. 132, 272-281.

Mia, M.A.B., Shamsuddin, Z.H, Zakaria, W. and Marziah, M. (2005) High-yielding and quality banana production through plant growth promoting rhizobacterial inoculation. Fruits, 60,179-185.

Mia, M.A.B., Shamsuddin, Z.H, Zakaria, W. and Marziah, M. (2007) Associative nitrogen fixation by Azospirillum and Bacillus spp. in bananas. Infomusa, 16 (1\&2), 11-15.

Moawad, H. and Abd El-Rahim, Wafaa M. (2002) Assessment of symbiotic performance of several bean cultivars inoculated with their specific rhizobia. Egyp. J. Microbiol. 37(3), 247-262 .

Moawad, H., Abd El-Rahim, Wafaa M. and Abd El-Aleem, D. (2004) Performance of Phaseolus bean rhizobia in soils from the major production sites in Nile Delta. $J$. Comptes rendus - Biologies, 327(5), 445-453. 
Monibe, M., Emam, N. F., Makboul, H. E. and Bedaiwi, E. H. (1998) Comparative study on the effect of different carbon and nitrogen sources on IAA production by Pseudomonas sp. Proceedings of the Second Conference of the Agricultural Development Research, Cairo. II. Food Science and Microbiology. Undated, pp. 275-286.

Mozingo, R.W., Coffelt, T.A. and Wright, F.S. (1991) The influence of planting and digging dates on yield. Value and grade of four Virginia-type peanut cultivars. Peanut Sci. 18, 55-63.

Naab, B.J., Tsigbey, K.F., Prasad, P.V.V., Boote, J.K., Balley, E.J. and Brandenbrg, L.R. (2004) Effects of sowing date and fungicide application on yield of early and late maturing peanut cultivars grown under rainfed conditions in Chana. Crop Prot. 24, 325-332.

Nautiyal, C.S. (1999) Inefficient microbiological growth medium for screening phosphate solubilizing microorganisms. FEMS Microbiology Letters. 170, 265- 270.

Rizzolo, L. J. and Joshi, H. C. (1993) Apical orientation of the microtubule organizing center and associated $\gamma$-tubulin during the polarization of the retinal pigment epithelium in vivo. Dev. Biol.157, 147-156.

Salem, E.M. (2008) Effect of irrigation water quantities, plant density and spraying with boron on sunflower growth and productivity under the New Valley conditions. $P h D$. Thesis, Fac. Agric. Sci., Ain Shams Univ., pp.9.

Sakhabutdinova, A.R., Fatkhutdinova, D.R., Bezrukova, M.V. and Shakirova, F.M. (2003). Salicylic acid prevents the damaging action of stress factor in wheat plants. Bulg. J. Plant Physiol., Special Issue: 314-319.

Scholhorn, R. and Burris, R.H. (1967) Acetylene as a competitive inhibitor of $\mathrm{N}_{2}$ fixation. Proc. Natl. Acad. Sci. USA, 58, 213-216.

Shu, Y. and Hui, H. L. (2008) Role of salicylic acid in plant abiotic stress. Zeit. Naturforsch. Section C, Biosciences, 63 (5/6), 313-320.

Sujatha, K.B. (2001) Effect of foliar spray of chemicals and bio-regulators on growth and yield of green gram (Vigna radiate L.). M.Sc. Thesis. Tamil Nadu Agric. Univ., Coibatore.p.22.

Usha, V. (2003) Production and partial characterization of antimicrobial proteases from Pseudomonus spp. Annals of Plant Protection Sciences, 17 (2), 602-608.

Van Rossum, D., Schuurmans, F. P., Gillis, M., Muyotcha, A., Van Verseveld, H. W. and Stouthamer, F. C. (1995) Genetic and phenetic analyses of Bradyrhizobium strains nodulating peanut (Arachis hypogaea L.) roots. Appl. Environ. Microbiol. 61, 1599-1609.

Vargas, P.A., Ho, W. C., Lim, M., Enz, S. and Aylett, R. (2009) To forget or not to forget "Towards a Rob ethical Memory Control", Proceedings of the AISB, Endinburgh, UK, pp. 18-23. 
Verma, J. P., Yadav, J. and Tiwari, K. N. (2010) Application of Rhizobium sp. BHVRCOL nodulation, Plant biomass and yields of chickpea (Cicer arietinum L.) Int. J. Agric. Res. 5, 148-156.

Wahid, A., Gelani, S., Ashraf, M. and Foolad, M.R. (2007) Heat tolerance in plants: An overview. Environmental and Experimental Botany, 61, 199-1033.

Wilson, D. O. and Reiesenauer (1963) Determination of leghaemoglobin in legume nodule. Analy. Biochem., 6, 27-30.

Wu, S.C., Cao, Z. H., Li, Z.G., Cheung, K.C. and Wang, M.H. (2005) Effects of biofertilizer containg $\mathrm{N}$-fzer, $\mathrm{P}$ and $\mathrm{K}$ solubilizers and AM fungi on maize growth: a greenhouse trial. Geoderma, 125(1-2),155

(Received 26/2/2013; accepted $2 / 6 / 2013)$

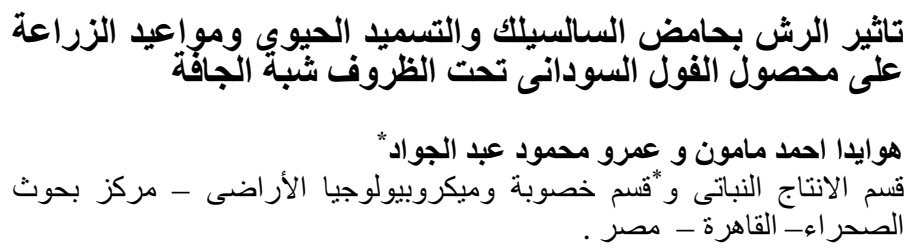

تم اجراء تجربتين حقليتين بالمزرعة البحثية بالخارجة التابعة لمركز بحوث المبرث

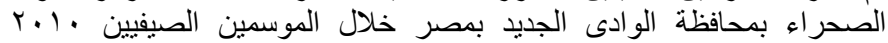

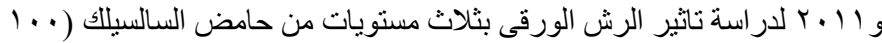

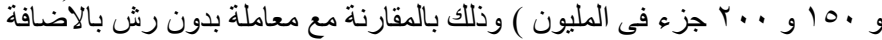

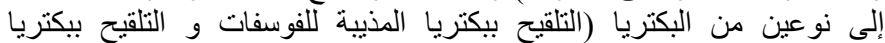

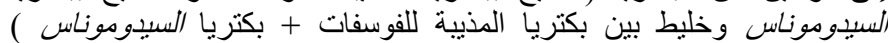

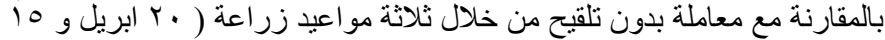

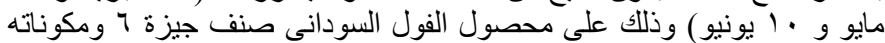

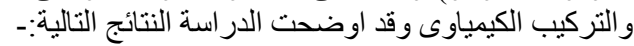

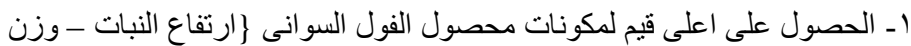

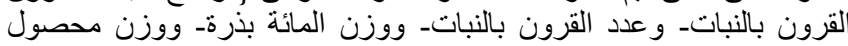

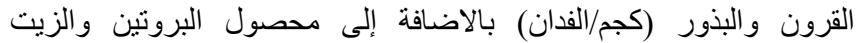

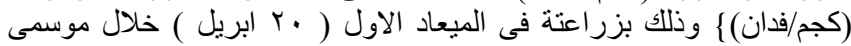

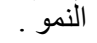

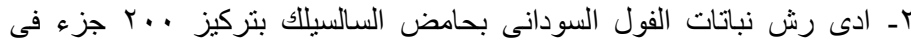

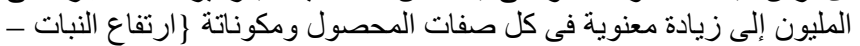

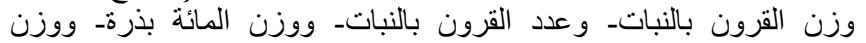

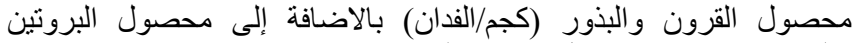
و الزيت (كجم/فدان) 
r- ادت معاملة الخلط بين التسميد الحيوى البكتريا الدذيبة للفوسفات وبكتريا

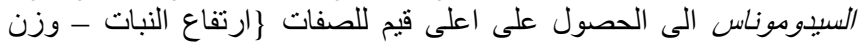

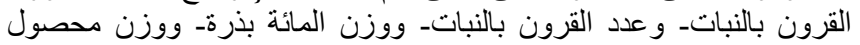

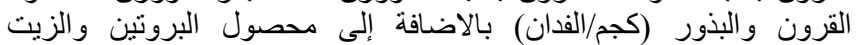

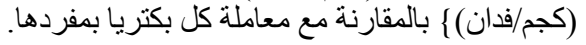

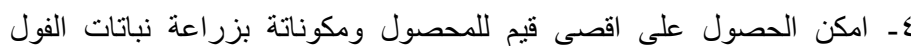

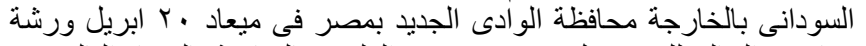

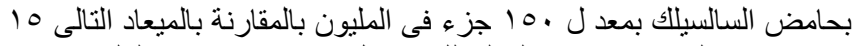

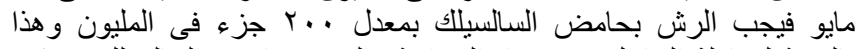

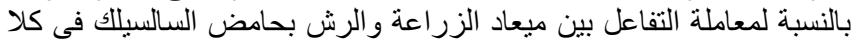
الموسميين.

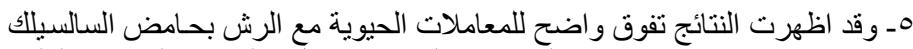

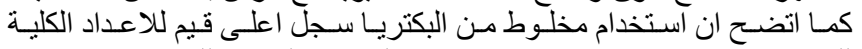

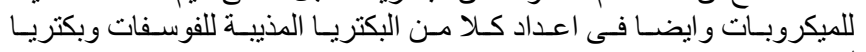

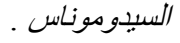

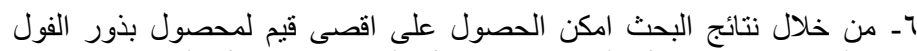

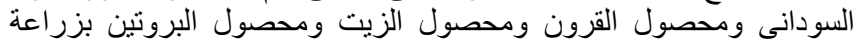

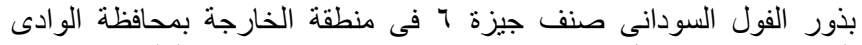

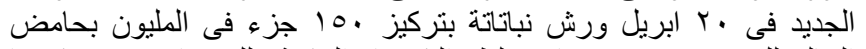

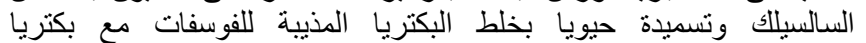

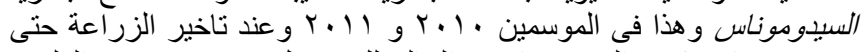

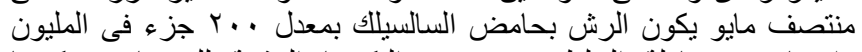
وايضا مع معاملة الخلط بين نوعى البكتريا المذيبة للفوسفات وبكتريا

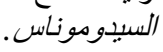

